\title{
A Review on the Traditional Chinese Medicinal Herbs and Formulae with Hypolipidemic Effect
}

\author{
Tung-Ting Sham, ${ }^{1}$ Chi-On Chan, ${ }^{1,2}$ You-Hua Wang, ${ }^{3}$ Jian-Mei Yang, \\ Daniel Kam-Wah Mok, ${ }^{1,2}$ and Shun-Wan Chan ${ }^{1,2,5}$ \\ ${ }^{1}$ Department of Applied Biology and Chemical Technology, The Hong Kong Polytechnic University, Hong Kong \\ ${ }^{2}$ State Key Laboratory of Chinese Medicine and Molecular Pharmacology (Incubation), Shenzhen 518057, China \\ ${ }^{3}$ Longhua Hospital, Shanghai University of Traditional Chinese Medicine, Shanghai 200032, China \\ ${ }^{4}$ Xuhui District Central Hospital of Shanghai, Shanghai 200031, China \\ ${ }^{5}$ Food Safety and Technology Research Centre, Department of Applied Biology and Chemical Technology, \\ The Hong Kong Polytechnic University, Hong Kong
}

Correspondence should be addressed to Daniel Kam-Wah Mok; bcdaniel@polyu.edu.hk and Shun-Wan Chan; bcswchan@polyu.edu.hk

Received 28 February 2014; Revised 4 May 2014; Accepted 10 May 2014; Published 7 July 2014

Academic Editor: Sharad S. Singhal

Copyright (C) 2014 Tung-Ting Sham et al. This is an open access article distributed under the Creative Commons Attribution License, which permits unrestricted use, distribution, and reproduction in any medium, provided the original work is properly cited.

Hyperlipidemia, characterized by the abnormal blood lipid profiles, is one of the dominant factors of many chronic diseases such as diabetes, obesity, and cardiovascular diseases (CVD). For the low cost, effectiveness, and fewer side effects, the popularity of using traditional Chinese medicine (TCM) to handle hyperlipidemia is increasing and its role in health care has been recognized by the public at large. Despite the importance of TCM herbs and formulations, there is no comprehensive review summarizing their scientific findings on handling hyperlipidemia. This review summarizes the recent experimental and clinical results of nine representative single Chinese herbs and seven classic TCM formulae that could improve lipid profiles so as to help understand and compare their underlying mechanisms. Most of single herbs and formulae demonstrated the improvement of hyperlipidemic conditions with multiple and diverse mechanisms of actions similar to conventional Western drugs in spite of their mild side effects. Due to increasing popularity of TCM, more extensive, well-designed preclinical and clinical trials on the potential synergistic and adverse side effects of herb-drug interactions as well as their mechanisms are warranted. Hyperlipidemic patients should be warned about the potential risks of herb-drug interactions, particularly those taking anticoagulants and antiplatelet drugs.

\section{Introduction}

Hyperlipidemia comprises a heterogeneous group of disorders, characterized by high levels in one or more lipids and/or lipoproteins [atherogenic free fatty acids (FA), triglycerides (TG) (hypertriglyceridemia), small dense low-density lipoprotein cholesterol (LDL-C) (hypercholesterolemia), and apolipoprotein (apo) B], and/or low level in antiatherogenic high density lipoprotein cholesterol (HDL-C), in the circulation [1-3]. In 2013, the American Heart Association reported that proportions of American adults aged 20 or above had abnormal blood lipid serum profile (details are shown in Table 1) [4]. These lipid disorders may occur in primary (inherited) [5-7] or secondary form [8]. Secondary hyperlipidemia arises from diet, alcohol intake, estrogen therapy, or diseases such as diabetes mellitus, hypothyroidism, erythematosus, and chronic renal diseases $[9,10]$. In fact, hyperlipidemia is one of the risk factors of CVD [11], which becomes one of the major killers around the world. It was projected that by 2030 there will be about 23.3 million CVD deaths worldwide [12]. Also, CVD has imposed great medical burden to different societies around the world. The global burden of CVD is beginning to be viewed as high as infectious diseases [13]. Apart from CVD, hyperlipidemia is also closely associated with diabetes, insulin resistance, and obesity [10]. Reduction of total cholesterols (TC) and LDL-C by dietary alterations and medications that affect lipid metabolism [14] is found to reduce the occurrence of atherosclerosis in 
TABle 1: Average percentage of abnormal blood lipid levels among Americans at age 20 or above [4].

\begin{tabular}{|c|c|c|c|c|}
\hline Blood lipid serum & \multicolumn{2}{|c|}{ Total blood cholesterol level } & LDL-C level & HDL-C level \\
\hline Abnormal level (mg/dL) & $\geq 200$ & $\geq 240$ & $\geq 130$ & $\leq 40$ \\
\hline Average percentage of Americans $\#$ & $\sim 42.4 \%$ men & $\sim 12.8 \%$ men & $\sim 34.4 \%$ men & 29.3\% men \\
\hline & $\sim 12.8 \%$ women & $\sim 13.6 \%$ women & $\sim 30.3 \%$ women & $\sim 12.6 \%$ women \\
\hline
\end{tabular}

\# Americans include non-Hispanic whites, non-Hispanic blacks, and Mexican-Americans at age 20 or above.

animals and clinical cardiovascular events in human [15]. Thus, prevention and treatment of hyperlipidemia are effective approaches to reduce the incident rate of chronic diseases.

Although Western medicines have been the dominant treatment used by hyperlipidemic patients, their adverse effects and part of patients' intolerance to the pharmacotherapy make traditional Chinese medicine (TCM) one of their alternatives. There is a growing interest in TCM treatment of hyperlipidemia. In the last few decades, hundreds of Chinese herbal medicines in the form of compounds, extracts, single herbs, or formulae have been reported to be effective for the prevention and treatment of hyperlipidemia [16], especially those high-fat diet (HFD) induced cases. In real practice, formulae (at least consist of two herbs) are commonly prescribed to patients. A formula generally is composed of four components: monarch, minister, assistant, and guide. The later three components aid the effects and facilitate the delivery of monarch (the principal component) or lower the toxicity of other components. Based on the patient's overall body situation, Chinese medicine practitioners always use classical formulae, rather than a single herb, as a foundation to modify the proportion and composition of the ingredients with addition or substitution by other herbs to prepare a specific formulation for individual patients.

Currently there are more than 50 TCM formulae in the form of patent drugs that have been approved by China Food and Drug Administration used for treating hyperlipidemia [16]. This implies that there is a great demand on hyperlipidemic TCM products in the market. This review summarizes TCM herbs and formulae that were proved to have effect in controlling blood lipid profiles in vivo, in vitro, and clinically so as to help people to understand and compare their underlying mechanisms. In this review, nine representative single herbs that have been studied extensively are selected (Table 2). Additionally, seven well-known TCM formulae are also covered here (Table 3 ).

\section{Normal Dietary Lipid Metabolism in the Circulation}

Lipid metabolisms involve different lipoproteins in the anabolism and catabolism of these substances (Figures 1 to 2) $[3,9,14,17,18]$. TG, phospholipids, and cholesterol esters (CE) are the predominant dietary lipids. These lipids, mainly TG, are hydrolyzed by different pancreatic lipases in the intestine and then absorbed by intestinal mucosal cells and secreted into mesenteric lymphatic vessels in the form of chylomicrons with apoB-48. The newly synthesized TG and $\mathrm{CE}$ in the chylomicrons are hydrolyzed by lipoprotein lipase (LPL) to yield chylomicron remnant particles which are cleared by LDL receptors (LDLR) and LDLR-related proteins to the liver. The liver secretes very low-density lipoproteins (VLDL) that contain specific apoB-100, apoC-II, and apoE that bind to enzymes or receptors to facilitate the lipid transfer to the peripheral tissues including vessels for metabolism or storage. ApoB-100 is the main apolipoprotein needed for LDL uptake by the liver. TG is hydrolyzed by LPL in VLDL which is further transformed into TG-reduced intermediate-density lipoprotein (IDL) followed by LDL. LDL is recirculated into the liver or peripheral tissues [19].

HDL plays a critical role in cholesterol homeostasis to induce antiatherogenic effect, which is brought by a process called "reverse cholesterol transport" $[2,20]$.

The process is the opposing movement of cholesterol from peripheral cells through plasma to the liver. The process involves removal of cholesterol from arterial macrophages and peripheral cells and delivery of the excess cholesterol to the liver for excretion. HDL, containing apoA-1 and other enzymes, is synthesized by the liver in a cholesterol deplete state and takes up excess cellular cholesterol from peripheral cells and arterial macrophages. The excess cholesterol is then delivered to steroidogenic organs for hormone synthesis, or to the liver by binding to scavenger receptor class $\mathrm{B}$, type I (SRBI) for further elimination into the bile as free cholesterol or as biliary acids after metabolism [21]. Liver X receptor (LXR) and farnesoid X receptor (FXR) maintain cholesterol, bile acid, and TG homeostasis [22]. This helps the removal of nonesterified free cholesterol from the blood circulation, thereby preventing the formation of arterial plaques. In indirect reverse cholesterol transport, $\mathrm{CE}$ is exchanged $1: 1$ for TG between apo B-containing lipoproteins (chylomicrons, VLDL, IDL and LDL) and $\mathrm{HDL}_{2}$ in a process regulated by the cholesterol ester transfer protein (CETP). In addition to the cholesterol removal, HDL also exerts other antiatherogenic and vascular protective functions [2] such as antioxidative $[23,24]$, antithrombotic [25], and anti-inflammatory actions [26].

Hence, the HDL-C level correlates inversely with the risk of CVD and atherosclerosis while high level of LDL-C highly increases their risk.

\section{Lipid Abnormality in Hyperlipidemia Resulting in Atherosclerosis}

Lipid abnormality in hyperlipidemia is an increase in nonesterified free FAs in the circulation, an inadequate esterification, and/or a reduced free FA metabolism [27]. The reduced retention of FAs by adipose tissue due to excessive visceral adiposity leads to an increased flux of free FAs 
TABLE 2: A summary of antihyperlipidemic effects of representative Chinese herbal medicines.

\begin{tabular}{|c|c|c|c|}
\hline $\begin{array}{l}\text { Chinese herbal } \\
\text { medicines }\end{array}$ & Sources & $\begin{array}{l}\text { Possible bioactive } \\
\text { compounds }\end{array}$ & $\begin{array}{l}\text { Effects mentioned in TCMs } \\
{[54,94]}\end{array}$ \\
\hline $\begin{array}{l}\text { Alismatis Rhizoma } \\
\text { (Zexie) }\end{array}$ & $\begin{array}{l}\text { Dried rhizome of } \\
\text { Alisma orientale } \\
\text { (Sam.) Juzep. }\end{array}$ & $\begin{array}{l}\text { Alisol-triterpenes } \\
{[59]}\end{array}$ & $\begin{array}{l}\text { (i) Promoting urination to } \\
\text { drain dampness } \\
\text { (ii) Discharging heat } \\
\text { (iii) Resolving turbidity and } \\
\text { lowering lipid }\end{array}$ \\
\hline
\end{tabular}

$\begin{array}{ll} & \begin{array}{l}\text { Dried rhizome of } \\ \text { Coptis chinensis }\end{array} \\ \text { (Huanglian/Goldthread) } & \begin{array}{l}\text { Franch. or } \text { C. } \\ \text { deltoidea C. Y. } \\ \text { Cheng et Hsiao. }\end{array}\end{array}$

Dried rhizome of Cheng et Hsiao.
Alkaloids [64], esp.

(i) Clearing heat and drying

(ii) Discharging fire and removing toxin

Possible hypolipidemic mechanisms

(i) Decreasing the liver synthesis of cholesterol [57]

(ii) Lessening lipid peroxidation and activating antioxidant enzymes [58]

(i) Reducing lipid peroxidation

[63, 64]

(ii) Upregulating PPAR $\alpha$

(iii) Negative modulation of FXR to upregulate the gene expression of CYP7Al for cholesterol conversion into bile acids [64]

(iv) Decreasing degradation of dietary polysaccharides [65]

(v) Upregulation of LDLR in vitro and in vivo [66]

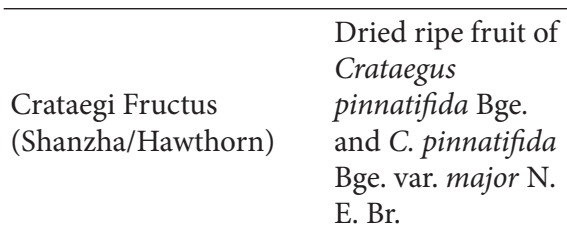

Polyphenols [70] (i) Promoting digestion and and triterpenic invigorating the stomach acids for example oleanolic acid and ursolic acid [71]

(ii) Resolving turgidity and lowering lipid

(iii) Moving Qi and dissipating blood stasis

(i) Greatly tonifying the original $Q i$

$\begin{array}{ll}\text { (i) Saponins } & \text { (ii) Resuming pulse and } \\ {[78,79]} & \text { securing collapse }\end{array}$ $[78,79]$

Ginseng Radix et Rhizoma

(Renshen/Asian ginseng)

Dried root of Panax ginseng C. A. Mey.

\section{(ii) Acidic} polysaccharides [81] (iii) Phenolic extract [83] (iii) Replenishing "lung" and "spleen"

(iv) Engendering fluid and nourishing blood

(v) Calming the mental state and enhancing intelligence

(i) Activating PPAR $\alpha$ [69]

(ii) Inhibiting intestinal ACAT activity in human colon carcinoma cell line Caco-2 [71]

(i) Improving lipid peroxidation in liver by reduction in serum MDA $[76,83,87]$

(ii) Activating LPL activity [81] (iii) Inhibiting pancreatic lipase [82] (iv) Inhibiting food appetite via modifying the serum content and mRNA expression of neuropeptide Y, Y2 receptor, and peptide YY [85]

(i) Reducing HMG-CoA reductase (ii) Reducing lipid peroxidation by increase in the activity of antioxidant hepatic SOD and glutathione peroxidase [95] (iii) Inducing the biosynthesis of bile acids from cholesterol and promoting the $\beta$-oxidation of FA in the liver [96]

(iv) Acting as a dual FXR/LXR $\alpha$ agonist [124]

\begin{tabular}{lll}
\hline & Washed and & \\
Oryzae cum Monasco & $\begin{array}{l}\text { cooked rice } \\
\text { Semen (Red yeast }\end{array}$ & $\begin{array}{l}\text { Monacolins, esp. } \\
\text { monacolin K }\end{array}$ \\
rice/Hongqu) & $\begin{array}{l}\text { Monascus } \\
\text { purpureus Went }\end{array}$ & {$[104,110]$} \\
\hline
\end{tabular}

Puerariae Lobatae Radix Dried root of (Gegen/Kudzu root) Pueraria lobata Puerarin [113] (i) Strengthening "spleen" to improve food digestion (ii) Promoting blood circulation to dissipate blood stasis

(i) Resolving the flesh and reducing fever

(ii) Engendering fluid to quench thirst

(iii) Promoting eruption

(iv) Uprasing the middle $Q i$ to relieve diarrhea

(v) Unblocking meridian and activating collaterals

(vi) Removing wine toxin
Containing a family of monacolins that resemble HMG-CoA reductase inhibitors [104] (i) Promoting cholesterol and bile acids excretion in liver [113] (ii) Estrogen-like effect on lipid metabolism in liver and adipose tissues [116]

(iii) Hepatoprotective effect in OVX-induced hepatic steatosis [114] 
TABLE 2: Continued.

\begin{tabular}{|c|c|c|c|c|}
\hline $\begin{array}{l}\text { Chinese herbal } \\
\text { medicines }\end{array}$ & Sources & $\begin{array}{l}\text { Possible bioactive } \\
\text { compounds }\end{array}$ & $\begin{array}{l}\text { Effects mentioned in TCMs } \\
{[54,94]}\end{array}$ & Possible hypolipidemic mechanisms \\
\hline $\begin{array}{l}\text { Rhei Radix et Rhizoma } \\
\text { (Dahuang) }\end{array}$ & $\begin{array}{l}\text { Root and rhizome } \\
\text { of Rheum } \\
\text { palmatum L., } R . \\
\text { tanguticum Maxim } \\
\text { or R. officinale } \\
\text { Baill. }\end{array}$ & Rhein [123-125] & $\begin{array}{l}\text { (i) Relaxing the bowels } \\
\text { (ii) Cooling the blood and } \\
\text { removing toxin } \\
\text { (iii) Expelling stasis to } \\
\text { unblock the meridian }\end{array}$ & $\begin{array}{l}\text { (i) Inhibitory effect on HMG-CoA } \\
\text { reductase [123] } \\
\text { (ii) Having LXR antagonism and } \\
\text { regulation of uncoupling protein-1 } \\
\text { expression in brown adipose tissues } \\
\text { [125] }\end{array}$ \\
\hline $\begin{array}{l}\text { Salviae Miltiorrhizae } \\
\text { Radix (Danshen) }\end{array}$ & $\begin{array}{l}\text { Dried root of } \\
\text { Salvia miltiorrhiza } \\
\text { Bunge. }\end{array}$ & $\begin{array}{l}\text { (i) Danshensu } \\
\text { (ii) Rosmarinic } \\
\text { acid } \\
\text { (iii) Salvianolic } \\
\text { acid A and B [133] }\end{array}$ & $\begin{array}{l}\text { (i) Promoting blood } \\
\text { circulation and dispelling } \\
\text { stasis } \\
\text { (ii) Nourishing the blood and } \\
\text { calming mental state } \\
\text { (iii) Regulating menstruation } \\
\text { and suppressing pains } \\
\text { (iv) Cooling blood and } \\
\text { eliminating carbuncle }\end{array}$ & $\begin{array}{l}\text { (i) Containing antioxidant (esp. } \\
\text { salvianolic acid B) for prevention of } \\
\text { endothelial damage and inhibition } \\
\text { of LDL oxidation [133] } \\
\text { (ii) Acting as a FXR/LXR } \alpha \\
\text { coagonist [134] }\end{array}$ \\
\hline
\end{tabular}

through the hydrolysis of adipocyte TG, returning to the liver, which stimulates hepatic TG synthesis at hepatocytes, promoting the production of apolipoprotein B and the assembly and secretion of TG containing VLDL. When plasma TG concentration subsequently increases, TG-rich HDL-apoA-1 particles are formed and undergo catabolism. Elevated VLDL particles due to hepatic overproduction are lysed and hence fail to bind efficiently to LDLR, while the exchange of CE with TG forms TG-rich lipoproteins, resulting in the formation of abnormal small dense LDL-C particles [28, 29]. These small dense LDL particles have weaker interaction with LDLR and stay longer than normal LDL in the blood so they readily penetrate through endothelial fenestrations to accumulate in the subendothelial space [30] where they are oxidized by reactive oxidative species (ROS) such as superoxide generated by endothelial cells [31] and activated leukocytes [32]. The oxidized LDL (ox-LDL) is a key component in the development of endothelial lesion $[33,34]$. It can induce endothelial expression and secretion of cytokines, growth factors, and several cell surface adhesion molecules that are capable of recruiting circulating monocytes and $\mathrm{T}$ lymphocytes into the intima.

Ox-LDL activates the differentiation of monocytes into macrophages. It also inhibits the production of vasodilators such as nitric oxide (NO) [35] released by the en-dothelial cells [36]. The ox-LDL is mostly taken up by macrophage scavenger receptors [37] and accumulates to become large foam cells which become fatty streak with $\mathrm{T}$ cells and smooth muscles. The fatty streak then progresses into an intermediate lesion, finally into a fibrous plaque which enlarges and projects into the arterial lumen and impede the flow of blood, thus inducing atherosclerosis. Thus, a strong association exists between LDL-C levelsand incidence of coronary artery diseases $[38,39]$. Figure 3 summarized the development of endothelial dysfunction.

\section{Western Medications}

There are over 10 conventional and new therapies for hyperlipidemia [10], such as agonists of peroxisome proliferator-activated receptors (PPAR) [40], inhibition of cholesterol absorption with statin, fibrates [41], ezetimibe [42], bile acid sequestrants [43], niacin [44, 45], and intake of omega-3 FA [46-48]. Statins and fibrates are the most commonly used lipid-lowering medications in primary and secondary preventions of atherosclerotic disease. Although these classical hypolipidemic agents are generally well tolerated, most of them have adverse effects [49]; they only target one class of lipoproteins or may not be affordable, deterring most patients from receiving treatments [50]. For instance, statin is a group of 3-hydroxy-3-methylglutaryl-coenzyme A (HMG-CoA) reductase inhibitors that inhibit the mevalonate pathway so as to suppress the production of cholesterol (Figure 1). Yet, statin was reported to have possible adverse effects (myalgia, fatigue, dyspnea, memory loss, and peripheral neuropathy) [51] and a number of patients did not respond well to such therapy [52]. If patients cannot tolerate the side effects of these hypolipidemic medicines, TCM will be an ideal alternative. It is because some of the TCM herbs and formulae have been demonstrated to be effective in handling hyperlipidemia, with low cost $[16,53]$. Figures $1-3$ indicate the potential mechanisms of TCMs and Western drugs reported.

\section{Studies of Single Herbs}

5.1. Alismatis Rhizoma. Alismatis Rhizoma, also known as Zexie in Chinese, is the dried rhizome of Alisma orientale (Sam.) Juzep. It is to promote urination to drain dampness, discharge heat, resolve turbidity, and lower lipid recorded in Pharmacopoeia of the People's Republic of China (CP) [54]. A number of Chinese published reports have demonstrated that AR is clinically effective in the treatment of hyperlipidemia. The oral administration of aqueous and alcoholic Zexie extracts $(6 \mathrm{~g} / \mathrm{kg} /$ day, 2 weeks) resulted in significant decreases in serum TG and TC levels in mice fed with $\operatorname{HFD}[55,56]$. However, the mechanism had not been clearly investigated. Dan. et al. found out that Zexie treatment $(2.26 \mathrm{~g} / \mathrm{kg} /$ day $)$ resulted in an obvious decrease in serum and liver cholesterol, TG level along with elevated serum HDL-C in 
TABLE 3: A summary of antihyperlipidemic effects of different TCM formulae.

\begin{tabular}{ccccc}
\hline TCM formulae & $\begin{array}{c}\text { Herbs (weight ratio in } \\
\text { dose if applicable) }\end{array}$ & $\begin{array}{c}\text { Effects mentioned in } \\
\text { TCMs }\end{array}$ & Effects on blood lipid profile & $\begin{array}{c}\text { Possible hypolipidemic } \\
\text { mechanisms }\end{array}$ \\
\hline
\end{tabular}

(i) Lowering serum TC and LDL-C

levels

$\begin{array}{lll}\text { Danggui-Buxue } & \begin{array}{l}\text { Astragali Radix and } \\ \text { Angelicae Sinensis Radix }\end{array} & \text { (i) Raising the } Q i \\ \text { decoction } & (1: 5)[153] & \text { blood [17] }\end{array}$
(ii) Increasing HDL-C level
(iii) No significant difference in TG
level as compared with diabetic

atherosclerosis model group

$[49,154]$

(i) A significant dose-dependent

decrease in free and esterified TC in the human monocyte derived macrophages in vitro [147]

$\begin{array}{lll}\text { Danshen-Gegen } & \text { Salviae Miltiorrhizae } & \text { (i) Promoting blood } \\ \text { formula } & \text { Radix and Puerariae } & \text { circulation } \\ & \text { Lobatae Radix }(7: 3)[53] & \text { (ii) Removing blood } \\ \text { stasis [200] }\end{array}$

(ii) Lowering TC and LDL-C levels mildly in patients suffering from

coronary artery disease compared with placebo [56]

(iii) Lowering TC and LDL levels in

postmenopausal women with

hypercholesterolemia [52]

\begin{tabular}{lll}
\hline & Curculiginis Rhizoma, & (i) Warming "kidney" \\
& Epimedii Folium, & Yang \\
Angelicae Sinensis & (ii) Nourishing "kidney" \\
& Radix, Morindae & Yin \\
Erxian & Officinalis Radix, & (iii) Clearing ministerial \\
decoction & Anemarrhenae & (iv) Harmonizing \\
& Rhizoma, and & thoroughfare and \\
& Phellodendri Chinensis & conception vessels \\
& Cortex (no fixed ratio) & (v) Balancing Yin-Yang \\
& {$[60]$} & {$[60]$} \\
\end{tabular}

(i) Suppressing serum TC and

LDL-C levels

(ii) No significant effect on HDL-C and TG levels in a menopausal rat model [54]
Downregulating the mRNA

expression of MCP-1, ICAM-1, and CD36 $[49,154]$
Dose-related suppression of acetylated LDL uptake by human macrophages [53] 
TABLE 3: Continued.

\begin{tabular}{lllll}
\hline TCM formulae & $\begin{array}{l}\text { Herbs (weight ratio in } \\
\text { dose if applicable) }\end{array}$ & $\begin{array}{c}\text { Effects mentioned in } \\
\text { TCMs }\end{array}$ & Effects on blood lipid profile & $\begin{array}{c}\text { Possible hypolipidemic } \\
\text { mechanisms }\end{array}$ \\
\hline & $\begin{array}{l}\text { Bupleuri Chinensis } \\
\text { Radix, Angelicae }\end{array}$ & & (i) Lowering serum TC and LDL-C & (i) Reversing energy and lipid \\
Sinensis Radix, & Rehmanniae Radix, & & levels \\
(ii) Increasing HDL-C level & (ii) Decreasing the
\end{tabular}

hyperlipidemic Kunming mice [57]. These results confirmed the efficacy of Zexie in the treatment of hyperlipidemia. They suggested that the herb might act by decreasing the liver synthesis of cholesterol, rather than by increasing the cholesterol catabolism. Zexie methanolic extract (150, 300, and $600 \mathrm{mg} / \mathrm{kg} /$ day, 6 weeks) also demonstrated the prevention of the oxidative stress by lessening lipid peroxidation and activating antioxidant enzymes and markedly decreased the serum and liver lipids in nonalcoholic fatty liver disease (NAFLD) rat induced by HFD [58]. Experiments showed that the active lipid-lowering compounds are triterpenoids. Supplement of the atherogenic diet with natural alisol A24-monoacetate isolated from Zexie in five concentrations (12.2-196.7 mg/kg/day, 10 days) lowered cholesterol levels in the plasma and liver of hypercholesterolemic rats in a dosedependent manner [59]. Other effective alisol-triterpenes included alisol A-23, 24-diacetate (derived chemically from alisol A), natural alisol C-23-monoacetate, alisol A, and alisol B-23-monoacetate isolated from Zexie [59]. Also, no adverse effects of triterpenoid-enriched extract of Zexie (360, 720 , and $1440 \mathrm{mg} / \mathrm{kg} / \mathrm{day})$ were observed in both genders of Sprague-Dawley (SD) rats after feeding for 90 days [60]. The adverse effects of Zexie are correlated with hepatotoxicity in chronic hepatitis $\mathrm{B}$ patients and nephrotoxicity following overdosage [61]. Alisol C, 16,23-oxido-alisol B, and alisol O in Zexie may cause nephrotoxicity [62].

5.2. Coptidis Rhizoma. Coptidis Rhizoma, commonly known as Huanglian in Chinese or Chinese Goldthread in English, is the dried rhizome of Coptis chinensis Franch. or $C$. deltoidea C. Y. Cheng et Hsiao. It is to clear heat and dry dampness, discharge fire, and remove toxin recorded in CP [54]. Different clinical, in vivo, and in vitro studies suggested multiple hypolipidemic mechanisms. Yokozawa et al. found that the herb water extract (50 and $100 \mathrm{mg} / \mathrm{kg} / \mathrm{day}$, 30 days) significantly reduced the levels of serum TC, LDL and ox-LDL, thiobarbituric acid-reactive substance, and liver cholesterol in a dose-dependent manner in Wistar rats but it did not reduce that of fecal cholesterol, suggesting that it reduced cholesterol synthesis by reducing lipid peroxidation [63]. Cao et al. got a consistent result as Yokozawa's but used the alkaloid extracts in HFD-fed SD rats instead [64]. The extracts $(50,100$, and $200 \mathrm{mg} / \mathrm{kg} /$ day $)$ markedly increased the level of liver total bile acid in a dose-dependent manner compared with simvastatin, which was attributed to the positive regulation of PPAR $\alpha$ and the negative modulation of FXR to upregulate the gene expression of CYP7A1 to increase its activity in the liver for cholesterol conversion into bile acids [64]. In addition, a new finding suggested that antimicrobial activities of the herb ethanol extract and berberine in HFD-fed C57BL/6J mice might also result in decreasing degradation of dietary polysaccharides, lowering potential calorie intake, and then systemically activating Fiaf protein and related gene expressions of mitochondrial energy metabolism in visceral adipose tissues [65]. Berberine is the main bioactive alkaloid of the herb. Oral administration of berberine $(0.5 \mathrm{~g}$ twice/day, 3 months) for the hypercholesterolemic Chinese patients resulted in significant decline in serum levels of cholesterol, TG, and LDL-C but no change in HDL-C compared to placebo groups [66]. This was also replicated by another study in human subjects with a mild weight loss (average $5 \mathrm{lb} /$ subject) and in SD rats [67]. The mechanism was explained by the upregulation of LDLR by berberine in the test of human hepatoma cells and the treatment of hyperlipidemic hamsters [66]. Combination of berberine with simvastatin increased the LDLR gene expression to a level significantly higher than that in monotherapies in rats and hypercholesterolemic patients [68].

Human subjects had no side effects observed and were well tolerated with the dosage of berberine $[66,67]$ or combined with simvastatin [68] so reduction of statin dosage in clinic by adding berberine may be possible.

5.3. Crataegi Fructus. Crataegi Fructus (the dried ripe fruit of Crataegus pinnatifida Bge. and C. pinnatifida Bge. var. major N. E. Br., known as Shanzha in Chinese or hawthorn in English) is a representative herb that is effective in promoting digestion and invigorating the stomach, resolving turgidity and lowering lipid, moving $Q i$ and dissipating blood stasis [54]. Studies have shown that the sugar-free water extract of Shanzha was effective against hyperlipidemia by activating PPAR $\alpha$ to lower lipid levels [69]. Apart from 


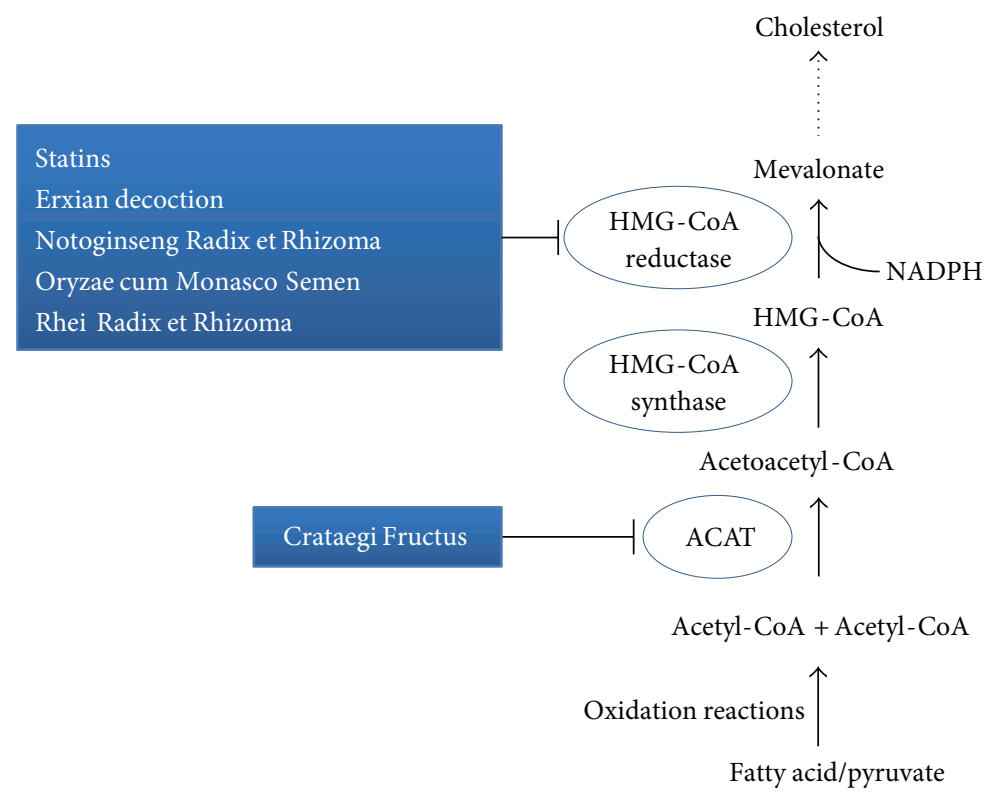

FIGURE 1: The simplified mevalonate pathway of cholesterol production. Potential therapeutic interventions in the pathway using conventional medications and TCMs are indicated. Dotted arrows: skipped pathway.

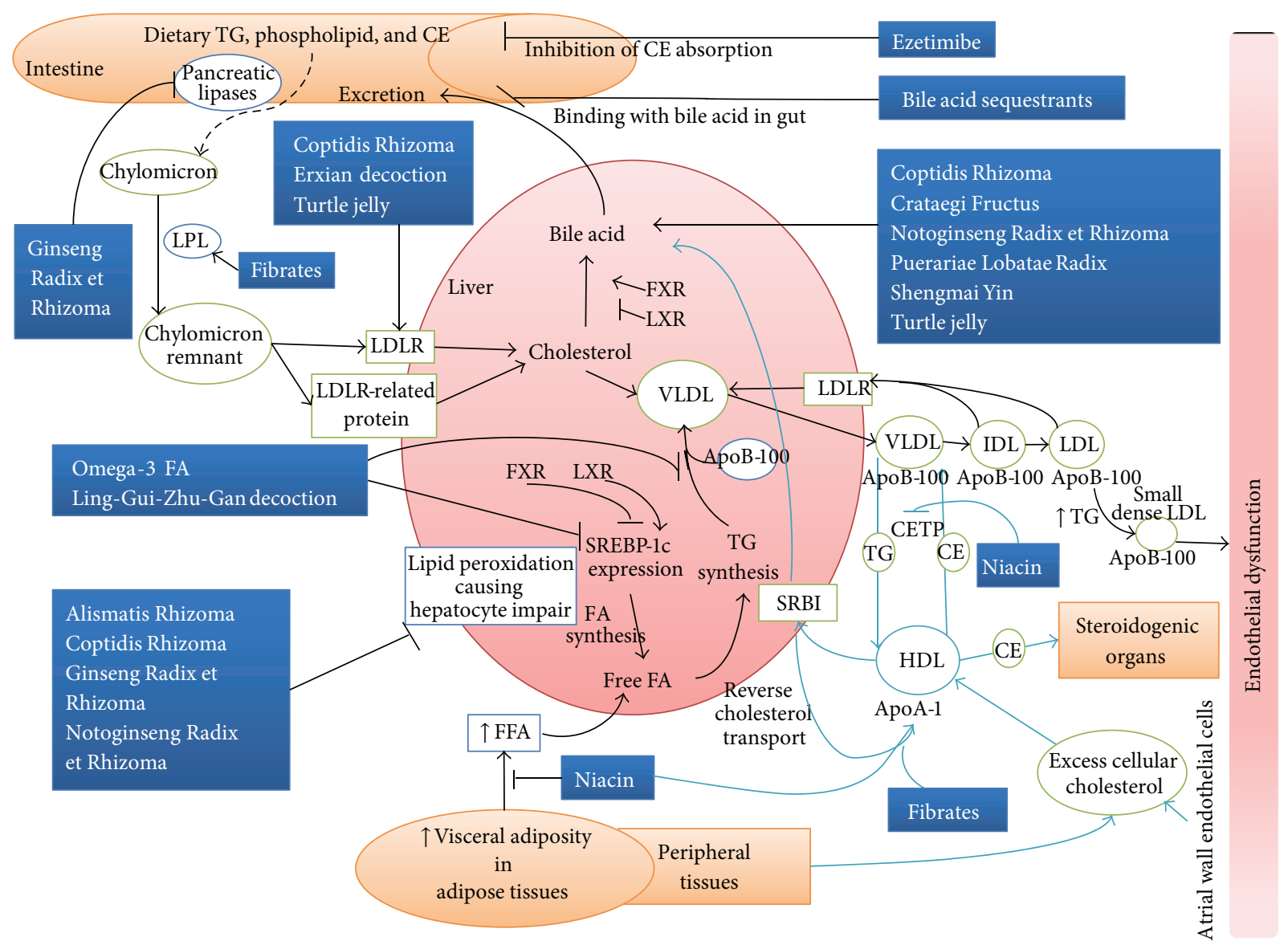

FIGURE 2: The primary pathways for the normal and abnormal metabolism of human plasma lipoproteins leading to endothelial dysfunction are summarized. Potential therapeutic interventions in the pathway using conventional medications and TCMs are indicated. Dotted arrows: skipped pathway. 


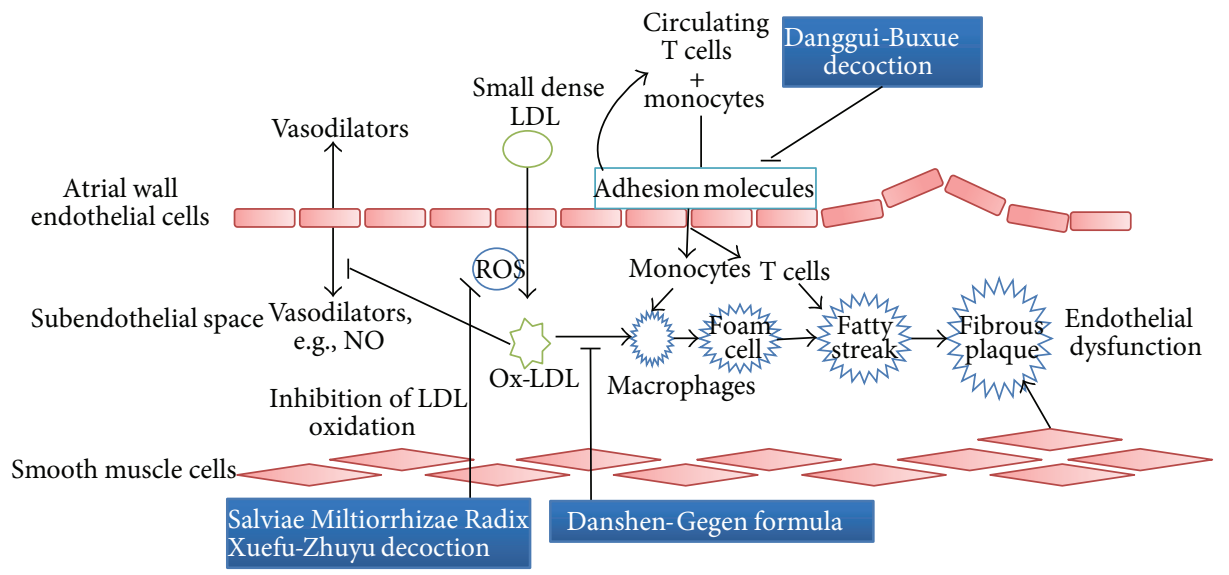

FIgURE 3: The endothelial dysfunction development is summarized and potential therapeutic interventions in the pathway using TCMs are indicated.

this, the extract also inhibited atherosclerosis progression by reducing inflammatory cytokine responses and regulating endothelial function in HFD-fed rats [70]. Shanzha is rich in triterpenic acids (e.g., oleanolic acid and ursolic acid) and polyphenols (such as epicatechin, procyanidins, hyperoside, isoquercitrin, and chlorogenic acid). Oleanolic acid and ursolic acid of Shanzha were found to be particularly responsible for lowering plasma VLDL and LDL cholesterol concentrations by inhibiting intestinal acyl CoA-cholesterol acyl-transferase (ACAT, the enzyme in the mevalonate pathway to synthesize cholesterol) activity in human colon carcinoma cell line Caco-2 [71]. The blunted acetylcholine-induced, endothelium-dependent relaxation of isolated aortas of HCD-fed rats was improved by dried powder of Shanzha crude drug $(20 \mathrm{~g} / \mathrm{kg} / \mathrm{day}, 4$ weeks) [72].

The most frequent mild adverse events were dizziness, nausea, fall, gastrointestinal haemorrhage, circulation failure, and erythematous rash $[73,74]$ but many cases had insufficient data supplied to prove the association between the herb and specific adverse effects [75]. The herb was well tolerated and there were no reports of drug interactions [75].

5.4. Ginseng Radix et Rhizoma and Ginseng Radix et Rhizoma Rubra. Ginseng Radix et Rhizoma (Renshen in Chinese and Asian ginseng in English) is the dried root of Panax ginseng C. A. Mey. As recorded in CP, it is used for greatly tonifying the original $\mathrm{Q} i$ resuming pulse and securing collapse, replenishing "lung" and "spleen," engendering fluid and nourishing blood, calming the mental state, and enhancing intelligence [54]. Ginseng Radix et Rhizoma Rubra, also called red ginseng, refers to the steamed and dried form of $P$. ginseng [54]. The lipid-lowering effect has been well studied in the last two decades in clinical trials $[76,77]$, in vivo [7885], and in vitro [86].

For the improvement of lipid profile, the $50 \%$ alcohol herb extract ( $2 \mathrm{~g}$ three times/day, 8 weeks) decreased TC, TG, and LDL levels and increased HDL in eight normal humans compared to the blood before the trial, which might be induced by antioxidant potential of the extract as one of action mechanisms [76]. The TC and LDL-lowering result was agreed on by a research with 20 hypercholesterolemic patients at a lower dose (100 $\mathrm{mg}$ twice/day, 8 weeks) [77]. Yet, the patients had no change of HDL, TG, or VLDL compared to control [77]. Red ginseng saponins $(0.01 \mathrm{~g} / \mathrm{kg}$ for 4 weeks) sustained LPL activity at a normal level or protected LPL activity, resulting in reduction of the rise in serum TG and TC in a cyclophosphamide-induced hyperlipidemia fasted rabbit model [78]. In addition to the saponins, acidic polysaccharides (100 to $1000 \mathrm{mg} / \mathrm{kg}$ ) isolated from Korean red ginseng using hyperlipidemic rats were also reported to have dose-dependently reduction in TG in serum and liver via the activation of LPL activity [81]. The hypercholesterolemiaenhanced platelet aggregation was also attenuated by Korean red ginseng, rich in glycosylated saponins $(200 \mathrm{mg} / \mathrm{kg} /$ day, 8 weeks) via the suppression of diacylglycerol liberation in rabbits fed on a diet high in cholesterol, but such effect was weaker than lovastatin [79].

The antioxidant properties of enzyme induced by the herb were also not consistent in humans $[76,87]$ and rabbit studies [83]. The improvement of lipid peroxidation in liver by reduction in serum malondialdehyde (MDA) was generally observed in most hypolipidemic studies $[76,83$, 87]. Apart from that, morphological changes of the aorta in hypercholesterolemic New Zealand white rabbits by the phenolic extract showed positive effects [83].

Weight gain reduction in 3\% herb extracted saponinscontaining HFD-fed male Balb/c mice may be mediated by pancreatic lipase inhibition [82]. Lin et al. suggested that intraperitoneal injections of ginsenoside $\mathrm{Rb}_{1}$ daily to C57BL/6 mice inhibited the desire for food intake via modifying the serum content and mRNA expression of neuropeptide Y, Y2 receptor, and peptide YY [85]. In the contrast, there were no significant body weight differences in hypercholesterolemic rabbits by the phenolic extract [83] and in HFD-fed C57BL/ 6 mice by fermented red ginseng powder $(150 \mathrm{mg} / \mathrm{kg} / \mathrm{day}, 11$ weeks) [88]. However, the combination 
of levan $(100 \mathrm{mg} / \mathrm{kg} /$ day $)$ and the fermented red ginseng powder $(150 \mathrm{mg} / \mathrm{kg} /$ day) significantly lowered body weight, serum TC and leptin levels, and fat mass with decreasing food efficiency ratio. This suggested improvement of leptin resistance associated with the obese mice. The disagreement in the hypolipidemic effect of the herb may be attributed to the composition of extract used in the experiments, administration method, dosages, and duration [83].

The safety profile of ginseng is generally good [89]. Excessive and uncontrolled intake of ginseng products may cause hypertension, nervousness, irritability, diarrhea, skin eruptions, and insomnia (collectively called ginseng abuse syndrome) [90]. More concerns about interactions between ginseng with anticoagulants, phenelzine [91], or warfarin [92] may increase the risk of bleeding episodes [93].

5.5. Notoginseng Radix et Rhizoma. Notoginseng Radix et Rhizoma is the rhizome and root of Panax notoginseng (Burk.) F. H. Chen, commonly called Sanqi in Chinese, is a herb that invigorates blood circulation and has been widely used in TCM to treat cardiovascular diseases [54, 94]. In CP, the action of Sanqi is to dissipate stasis, stanch bleeding, dispel swelling, and relieve pain [54]. Sanqi crude drug powder supplement incorporated in the HFD for SD rats $(10 \mathrm{~g} / \mathrm{kg}$, 4 weeks) was found to have improvement in lipid profiles, reduction of HMG-CoA reductase level, and inhibitory effect of lipid peroxidation by increase in the activity of antioxidant enzymes [hepatic superoxide dismutase (SOD) and glutathione peroxidase] [95]. Sanqi saponins including different ginsenosides and notoginsenosides were found to be the main active compounds. A systematic study [96] reported that diet induced hypercholesterolemic SD rats fed with total saponins of Sanqi (30 or $100 \mathrm{mg} / \mathrm{kg} / \mathrm{day}, 4$ weeks) could significantly reduce elevated serum TC, TG, LDL-C, and atherogenic index and increase HDL-C. The saponins significantly improved the endothelium-dependent vasodilatation, acetylcholine-induced NO production, and endothelial NO synthase mRNA expression. The findings suggested that Sanqi could prevent the development of hypercholesterolemia and atherosclerosis by inducing the biosynthesis of bile acids from cholesterol and promoting the $\beta$-oxidation of FA in the liver. Increasing the gene expression of endothelial NO synthase in endothelial cells and Sanqi antioxidative activity might bring about its vasoprotection. Another research also proved that the saponins $(100 \mathrm{mg} / \mathrm{kg} /$ day, 9 weeks) markedly reduced serum TC, TG, and blood viscosity in HFD-fed SD rats and exerted antiatherosclerosis through an anti-inflammatory action and regulation of the blood lipid profile, involving nuclear factor$\kappa \mathrm{B}$ signaling pathway [97]. Another experiment using the n-butanol extract (30, 60, and $100 \mathrm{mg} / \mathrm{kg} / \mathrm{day}, 4$ weeks) showed similar results and revealed that the extract acted as a dual FXR/LXR $\alpha$ agonist to prevent the accumulation of abnormal lipid in the hyperlipidemic rats [98]. One study [99] concerned that high fibrinogen is an emerging independent risk factor for cardiovascular diseases but statins could only slightly reduce blood fibrinogen level. In particular, intake of atorvastatin, a very potent lipid lowering agent, might have association with increase in fibrinogenaemia in some treated patients $[100,101]$. Compared to fluvastatin ( $3 \mathrm{mg} / \mathrm{kg} /$ day), Sanqi powder (43 and $86 \mathrm{mg} / \mathrm{kg} /$ day) showed a similar activity in decreasing plasma TC but more marked reduction in fibrinogenaemia in HFD-fed rats [99].

More awareness using the intravenous injection of Sanqi saponins should be taken since some adverse reactions such as epistasis, allergy, and even anaphylactic shock might be caused [102]. Abdominal complaints, nausea, and dyspepsia were observed clinically in a pellet consisting of extracts from Danshen, Sanqi, and Borneol [103].

5.6. Oryzae cum Monasco Semen. Oryzae cum Monasco Semen is commonly called as Hongqu in Chinese and red yeast rice (RYR) in English. It has long been recognized as a TCM to strengthen "spleen" to improve food digestion and promote blood circulation to dissipate blood stasis [94]. It is produced by solid-state fermentation of washed and cooked rice using red yeast (Monascus purpureus Went). RYR is well known for its blood cholesterol lowering effect. The fermentation of RYR produces a family of monacolins that resemble HMG-CoA reductase inhibitors [104]. Among these products, monacolin $\mathrm{K}$ is the same substance that the USA Food and Drug Administration approved as lovastatin [105]. Lots of evidences showed that RYR lowers cholesterol levels moderately compared to other statin drugs, but with less adverse effects [106-109]. One recent study demonstrated that 22 patients receiving RYR capsules that contained $7.2 \mathrm{mg}$ lovastatin and a total of $2.4 \mathrm{mg}$ of other monacolins experienced a significant reduction in LDL-C (23.0\%) and TC (15.5\%) compared to the placebo after 16 weeks of treatment [110]. Another randomized controlled trial showed that RYR preparations had lowering lipid effect, compared with placebo, statins, or other active lipid-lowering agents, and with no treatment received for 12 weeks [111]. In this test, the lipid modification effects of RYR were observed better than nicotinate and fish oils, similar to pravastatin, simvastatin, lovastatin, atorvastatin, or fluvastatin, but equal to or less effective than fenofibrate and gemfibrozil.

Dizziness and gastrointestinal discomfort were also reported in 1.3-36\% of participants after RYR intakes [111]. In spite of its therapeutic effect, the yeasts from the same genus of RYR (Monascus strains) could produce citrinin (a nephrotoxic mycotoxin) as a secondary toxic metabolite. A survey indicated that $69.0 \%$ of red yeast rice, $35.1 \%$ of dietary supplements, and $5.7 \%$ of red yeast rice processed products contained citrinin in Taiwan from 2009 to 2012 [112]. The safety control of this kind of products should be cautious.

5.7. Puerariae Lobatae Radix. Puerariae Lobatae Radix (Gegen in Chinese and Kudzu root in English) is the dried root of Pueraria lobata (Willd.) Ohwi for resolving the flesh and reducing fever, engendering fluid to quench thirst, promoting eruption, uprasing the middle $Q i$ to relieve diarrhea, 
unblocking meridian and activating collaterals, and removing wine toxin in CP [54]. For the hypolipidemic effect, puerarin, the major isoflavonoid compound of Gegen, $(300 \mathrm{mg} / \mathrm{kg} / \mathrm{day}$, 4 weeks), attenuated the increased TC induced by HCD in both serum and liver of SD rats. The cholesterol lowering action might be caused by the promotion of cholesterol and bile acids excretion in liver [113]. Additionally, this herb exhibited beneficial effect in lipid metabolism in ovariectomized (OVX) rats which imitated the postmenopausal situation of the disorders of lipid metabolism. The total isoflavones inhibited the increase in body weight and lipoprotein levels in OVX rats, which exhibited most of the characteristics of human menopausal symptoms compared to the OVX-control rats and exhibited a hepatoprotective effect in OVX-induced hepatic steatosis [114]. Long-term dietary Gegen extract supplementation containing $25.3 \%$ puerarin, $7.1 \%$ daidzin, and $0.8 \%$ daidzein (a polyphenol-free diet, with $0.2 \%$ kudzu root extract, 2 months) could improve blood glucose, lipid, and pressure control in intact and OVX stroke-prone spontaneously hypertensive rats [115]. The Gegen flavones (100 mg/kg/day, 5 weeks) demonstrated estrogen-like effect on lipid metabolism in liver and adipose tissue compared to estrogen-treated OVX Wistar rats [116].

However, the phytoestrogens of Gegen (coumestrol, genistein, and daidzein) may have potential interactions with the endocrine system, and therefore special attention should be drawn to the herbal formulae containing Gegen, especially the prescriptions for female patients at reproductive age suffering from respiratory diseases [117]. Also, a herb-drug interaction study pointed out that isoflavones and their glycosides and other polyphenols of Gegen might be transformed into conjugated metabolites in SD rats to compete with methotrexate (a drug for cancer treatment and may cause very serious, life-threatening side effects) to delay the elimination of methotrexate, increasing its life-threatening toxicity [118].

5.8. Rhei Radix et Rhizoma. Rhei Radix et Rhizoma (Dahuang in Chinese and rhubarb in English) comes from the root and rhizome of Rheum palmatum L., R. tanguticum Maxim, or $R$. officinale Baill. Dahuang has been widely used for the treatment of constipation, dysentery, and jaundice $[54,119-122]$. Rhein is an anthraquinone and is one of the major components of $R$. palmatum L. Rhein $(150 \mathrm{mg} / \mathrm{kg} /$ day) purified from $R$. tanguticum decreased the plasma levels of cholesterols, TG, LDL-C, and Apo-E, similar to the simvastatin group $(20 \mathrm{mg} / \mathrm{kg} / \mathrm{day})$ in $\mathrm{db} / \mathrm{db}$ diabetic mice, but it had a weaker inhibitory effect on HMG-CoA reductase than simvastatin in vitro [123]. Rhein $(150 \mathrm{mg} / \mathrm{kg} / \mathrm{day})$ was also found to decrease body and fat weight, lower hepatic lipid levels, improve insulin resistance, normalize alanine aminotransferase levels, and reverse hepatic steatosis on NAFLD in HFD-fed mice [124] and might protect against obesity and related metabolic disorders through LXR antagonism and regulation of uncoupling protein-1 expression in brown adipose tissues in HFD-fed mice [125]. Water extract capsules of root of $R$. officinalis
(50 mg/kg/day, oral ingestion with $200 \mathrm{~mL}$ of water for six months) was found to significantly lower TC and LDL-C levels but had no change in TG and HDL-C levels in the clinical trial group of patients with atherosclerosis compared to the control group [126]. The researchers suggested that the change aided the improvement in the endothelial function. The same type of water extract was administrated in New Zealand white rabbits and gained similar results [127]. The extract had antiatherosclerotic and plaque stabilizing effects which might occur due to a reduction of some inflammatory cytokines activated by toll-like receptors and nuclear factor $-\kappa \mathrm{B}$ signaling [127].

No serious adverse effects were observed throughout the clinical trial [126]. Only five patients in the trial group reported diarrhea, which was mild and resolved without symptomatic therapy [126]. Since Rhubarb has purgative effect due to its anthraquinones [128], the major symptoms of overdose are griping and severe diarrhoea with consequent losses of fluid and electrolytes [129].

5.9. Salviae Miltiorrhizae Radix. Salviae Miltiorrhizae Radix (also called Danshen in Chinese), the dried root of Salvia miltiorrhiza Bunge, is a TCM herb commonly used for the prevention and treatment of CVD. In CP [54], it is used for promoting blood circulation and dispelling stasis, nourishing the blood and calming mental state, regulating menstruation and suppressing pains, cooling blood, and eliminating carbuncle. It has been used for treatment of CVD for long time. The cardiovascular protective effect mainly contributed by Danshen's antioxidants has been observed in many animal models. Treatment with aqueous extract of Danshen $(600 \mathrm{mg} / \mathrm{kg} /$ day, 12 weeks) reduced TC and LDL-C with no change in TG and HDL-C and prevented formation of HFD induced fatty liver in an OVX hyperlipidemic rat model [130]. Oxidative stress is one of the causative factors that link hypercholesterolemia with the pathogenesis of atherosclerosis. Increased oxidative stress, resulting from increased ROS production, appears to play an important role in hypercholesterolemic atherogenesis [131]. The high antioxidative capacity of the Danshen aqueous extract may facilitate the removal of ROS in the circulation. Another study suggested that this antioxidant capacity and cholesterol lowering effect observed in the hypercholesterolemic restenosis New Zealand white rabbit model fed with $80 \%$ ethanol extract of Danshen treatment $(4.8 \mathrm{mg} / \mathrm{kg} /$ day, 6 weeks $)$ enhanced smooth muscle apoptosis and attenuates neointimal hyperplasia [132]. Same types of model fed with a HCD containing $5 \%$ water-soluble Danshen extract for 12 weeks reduced the atherosclerotic area in the abdominal aorta by $56 \%$ and cholesterol deposition in the thoracic aorta by $50 \%$, although it was weaker than probucol treated group. It concluded that Danshen's cholesterol-lowering effect and more importantly, the antioxidant, salvianolic acid $\mathrm{B}$, that accounted for about $75 \%$ of antioxidant activity in Danshen contributed to the prevention of endothelial damage and inhibition of LDL oxidation [133]. In another study, hyperlipidemic SD rats were treated with purified aqueous extracts of Danshen that contained Danshensu, rosmarinic acid, and salvianolic 
acid $A$ and salvianolic acid $B(50,100$, and $150 \mathrm{mg} / \mathrm{kg} /$ day, 4 weeks). They also decreased TC and TG levels and increased HDL-C serum levels, but this improvement likely contributed to the extract acting as a FXR/LXR $\alpha$ co-agonist [134].

There was low or nontoxic acute $(32 \mathrm{~g} / \mathrm{kg}$, twice/day) or subchronic administrations $(5.76 \mathrm{~g} / \mathrm{kg} /$ day, 13 weeks) of Danshen injection in rats but a significant decrease in TG and increase in total bilirubin [135]. Coadministration of Danshen may exaggerate the anticoagulant response to warfarin since Danshen might increase the bioavailability of warfarin $[136,137]$. Therefore, Danshen or its products such as Danshen-Gegen formula or Danshen dropping pills should be used under close medical supervision by people taking similar anticoagulants.

\section{Studies of TCM Formulae}

6.1. Danggui-Buxue Decoction. Danggui-Buxue decoction (DGBX) is a very common TCM prescription consisting of Astragali Radix and Angelicae Sinensis Radix at a dose of 1:5 [138]. In CP, its product "Danggui Buxue Koufuye" (a DGBX oral solution) is recorded to raise the $Q i$ and nourish the blood [54]. A study used DGBX (3 or $6 \mathrm{~g} / \mathrm{kg} /$ day) for 4 weeks to be orally administered to the diabetic atherosclerosis rats, which were induced by NO inhibition plus HFD [139]. Although there was no significant change in TG level, serum TC and LDL-C were significantly lowered, and the HDL-C was higher in the DGBX-treated group than that in diabetic atherosclerosis model groups. Treatment with DGBX in early diabetic atherosclerosis rats resulted in significant inhibition of mRNAs expression of monocyte chemoattractant protein1 (MCP-1), intercellular adhesion molecule-1 (ICAM-1), and CD36 (a FA translocase that mediates oxidized LDL uptake) in aorta so it helped the prevention of diabetic atherosclerosis. Another study showed the similar effect of DGBX $(1.68,8.4$ and $16.8 \mathrm{~g} / \mathrm{kg} /$ day) for 5 weeks but had obvious decrease in TG in all dosages [140]. DGBX (equivalent to $6 \mathrm{~g} / \mathrm{kg} / \mathrm{day}, 6$ weeks) also demonstrated improvement in hepatic lipid peroxidation by increase in SOD activities and decreased MDA levels in fibrotic livers of HFD-fed rats [141].

The clinical treatment of DGBX preparations at 1.5, 3.0 , or $6.0 \mathrm{~g} /$ day, 12 weeks was well tolerated, with no serious adverse events noted in postmenopausal women but no significant lipid profile changes were observed [142].

6.2. Danshen-Gegen Formula. Danshen-Gegen formula (DG), composed of Salviae Miltiorrhizae Radix and Puerariae Lobatae Radix, are traditionally paired clinically to treat atherosclerosis, myocardial infarction, and other cardiac symptoms and have been widely studied in the aspect of cardiovascular effects [143-146]. Various concentrations $(0.1-1.0 \mathrm{mg} / \mathrm{mL})$ of the water extract in the ratio of $7: 3$ were tested in the human monocyte derived macrophages loaded with acetylated LDL [147]. Compared to the control, the herbal mixture induced a significant dose-dependent decrease in the (free and esterified) cholesterols in the macrophages. However, the herb pair also induced an increase in ICAM-1 expression and monocyte adhesion at higher concentrations [147].

In a clinical trial, DG treatment ( $3 \mathrm{~g} /$ day, 24 weeks) in double-blind parallel fashion in patients suffering from coronary artery disease showed mild decrease in TC and LDL-C [148]. Another clinical research with postmenopausal women treated by the herb pair (two capsules, containing $1 \mathrm{~g}$ water extract/day) demonstrated that the herb-pair treated group has a significant improvement in intima-media thickness and a remarkable decrease in TC and LDL as compared to placebo-treated group after 12-month treatment [149]. For the genomic study [149] the herbal-treated group has a higher number of differential gene expressions identified as compared to the placebo-treated groups. It was suggested that the herb pair could diminish the process of cardiovascular deterioration in postmenopausal women.

For the safety of DG, its treatment in patients suffering from coronary artery disease was well tolerated [148]. Yet, coadministration of DG with anticoagulant warfarin or aspirin, respectively, can decrease the prothrombin time of the drugs and cause other significant pharmacokinetic and pharmacodynamic herb-drug interactions in SD rats [150].

6.3. Erxian Decoction. Erxian decoction (EX), designed by Zhang Bo-Na in early 1950s, consists of Curculiginis Rhizoma, Epimedii Folium (monarch), Angelicae Sinensis Radix, Morindae Officinalis Radix (minister), Anemarrhenae Rhizoma, and Phellodendri Chinensis Cortex (assistant) without fixed ratio [151]. It has long been used in TCM to treat Yang and Yin deficiency of "kidney," menopausal symptoms, and osteoporosis $[152,153]$. A study demonstrated that water extract of EX (equivalent to $4.10 \mathrm{~g}$ crude $\mathrm{drug} / \mathrm{kg} / \mathrm{day}, 8$ weeks) had hypolipidemic effects in a menopausal rat model but this was not observed in ethyl acetate $(0.11 \mathrm{~g} / \mathrm{kg})$, nbutanol $(0.470 \mathrm{~g} / \mathrm{kg})$, and the aqueous remaining fractions $(2.34 \mathrm{~g} / \mathrm{kg})$ of the water extracts [154]. Premarin, which is a conjugated estrogen for the replacement of hormone of postmenopausal women, did not show such effect. The serum levels of TC and LDL-C of EX groups were suppressed, possibly through the downregulation of HMG-CoA and upregulation of the LDLR while HDL-C and TG levels had insignificant change. Compared to the premarin, EX containing hypolipidemic components may have the advantage to hyperlipidemic postmenopausal women.

In a clinical trial, the water extract of EX (equivalent to $62 \mathrm{~g}$ of crude herbal materials/day, 12 weeks) is well tolerated, with no serious adverse events noted in Hong Kong perimenopausal women [153].

6.4. Ling-Gui-Zhu-Gan Decoction. Ling-Gui-Zhu-Gan decoction (LGZG) is an ancient Chinese herbal formula from "Jin-Gui-Yao-Lue" for warming Yang for resolving fluid retention and strengthening "spleen" to resolve dampness [155]. It consists of four herbs only: Poria, Cinnamomi Ramulus, Atractylodis Macrocephalae Rhizoma, and Glycyrrhizae Radix at the weight ratio of $4: 3: 3: 2$ [155]. Caloric restriction 
therapy has been studied with metabolic disorders such as type 2 diabetes $[156,157]$ and obesity [158] to normalize metabolism. Recently, scientists have studied caloric restriction therapy supplemented with LGZD in Wistar rats [155]. Although there were no statistical differences in blood lipid level between fasting and those fed with LGZD groups, the blood level of ghrelin, a starvation hormone [159], was lower in HFD rat groups fasting 24 hours intermittently supplemented with LGZD than those without LGZD. Thus, it was suggested that the decoction may help regulate appetite of the fasting rats and so the same may happen in human patients for easier completion of the caloric restriction therapy when they feel less hunger [155]. Another research group provided evidence on the traditional treatment principle of LGZD using HFD induced rat models of NAFLD [160]. The possible mechanisms of LGZG to regulate lipid metabolism are to increase serum thyroid hormone levels and improve FA synthesis via modulation of thyroid hormone receptor $\beta 1(\mathrm{TR} \beta 1)$ and carnitine palmitoyltransferase-1A (CPT1A) expression in liver and enhance metabolism and transport of FA through modulation of sterol regulatory element-binding protein 1c (SREBP-1c), long-chain acylCoA synthetase (ACSL), and ApoB-100 expression. The combination of Poria and Ramulus Cinnamomi might be crucial in LGZG to warm Yang to activate $Q i$ by increasing serum thyroid hormone levels and hepatic TR $\beta 1$ and CPT1A expression and enhancing $\beta$-oxidation of FA, so as to relieve water retention while Atractylodis Macrocephalae Rhizoma and Glycyrrhizae Radix assist such effect of warming Yang.

6.5. Shengmai Yin. Shengmai Yin (SMY) which is also called "Shengmai San" or "Pulse-activating decoction" is comprised of Ginseng Radix et Rhizoma, Ophiopogonis Radix, and Schisandra Chinensis Fructus $(2: 1: 2)$ [161], for treating cardiovascular diseases such as coronary heart diseases [162] and brain impairment [163]. It was first documented in "NeiWai-Shang-Bian-Huo-Lun" in the 13th century. Its patent drug "Shenmai Capsule" and SMY are officially recorded in CP with Ginseng Radix et Rhizoma Rubra replacing unprocessed Ginseng Radix et Rhizoma instead to replenish both $Q i$ and Yin energies, generate body fluids, and restore the pulses [54]. Some ingredients in SMY may increase the dissolution of schisandrin when decocting in vitro and delay its elimination and enhance its bioavailability in rat compared to the Schisandra Chinensis Fructus aqueous extract [164]. An animal study in a Chinese journal showed that Shengmaidan chewable tablets (equivalent to $2.0 \mathrm{~g}$ crude drug/kg, 4 weeks) could reduce TC and LDL-C in SD rat serum obviously while low dosage group (equivalent to $1.0 \mathrm{~g}$ crude drug/kg) could reduce TG in rat serum obviously [165]. It also decreased low-shear reduced viscosity of whole blood, plasma viscosity, haematocrit, and erythrocyte aggregation index but exhibited insignificant effect on the index of erythrocyte deformability. In another research, SMY had no significant effect on plasma lipids of Wistar rats but groups fed with HCD plus 4\% SMY for four weeks had lower hepatic cholesterol and TG contents [166]. SMY had no effect on fecal cholesterol excretion but higher fecal bile acid content was observed after the SMY treatment. Increased fecal bile acid excretion (the major degradation of endogenous cholesterol process) might stimulate the biosynthesis of bile acid using cholesterol as the precursor, resulting in an increase in hepatic cholesterol catabolism and so reducing hepatic cholesterol and thereafter TG accumulation.

No adverse reactions were found in clinical trials of SMY capsule [167] or injection to patients with coronary heart disease complicated with diabetes mellitus [168] which is consistent with the review [169]. Yet, a case of a 71-year-old man indicated that an adverse interaction between warfarin and SMY resulted in cerebral bleeding [170]. Our previous mentioned ginseng, one of the main ingredients in SMY, probably contributed to the interaction. Another ingredient Schisandra Chinensis Fructus might also increase the metabolism of the coadministered warfarin [171].

6.6. Turtle Jelly. Turtle (tortoise) jelly, also called Gui-linggao in Chinese, is a popular traditional functional food in Southern China to clear heat, remove toxin, and promote urination [172]. The main ingredient is Testudinis Plastrum (tortoise shell), plus various Chinese medicinal herbs such as Smilacis Glabrae Rhizoma, Millettiae Speciosae Radix, Mesonae Chinensis Herba, and Lonicerae Japonicae Flos [173]. The plasma of diet-induced hypercholesterolemic rats fed with turtle jelly ( 3.3 or $10 \mathrm{~mL} / \mathrm{kg} /$ day) for 30 days showed hypercholesteroleamic effect in terms of lowering the level of the serum TC and LDL, but increasing HDL level with a dosedependent improvement on the atherogenic index [173]. These also brought the protection of endothelial dysfunction and livers. The hypercholesterolemic effect was suggested to be related to the blockage of downregulation of LDLR and phosphoenolpyruvate carboxykinase (PEPCK) mRNA and protein expressions as well as suppression of the upregulation of PPAR $\alpha$ mRNA and protein expressions in the livers in rats fed with HCD.

There are no studies reporting its safety issue. Since the content of turtle jelly is very complicated, its long-term efficacy and safety still need further investigations.

6.7. Xuefu-Zhuyu Decoction. Xuefu-Zhuyu decoction (XFZY) was described in an ancient book called "Yi-LinGai-Cuo" in early 18th century [174] and is composed of two classic formulae (Taohong-Siwu decoction and Sini decoction): Bupleuri Chinensis Radix, Angelicae Sinensis Radix, Rehmanniae Radix, Paeoniae Rubra Radix, Carthami Flos, Persicae Semen, Aurantii Fructus Immaturus, Glycyrrhizae Radix, Platycodi Radix, Chuanxiong Rhizoma, and Achyranthis Bidentatae Radix [175]. It is a usual TCM formula to treat blood stasis in the chest region [176]. CP records its proprietary drug "Xuefu ZhuYu Jiangnang" (a XFZY oral capsule) for promoting blood circulation to remove blood stasis, moving $Q i$ to relieve pain [54]. Its blood lipid lowering effect and improvement of $Q i$ stagnation and 
blood stasis syndrome have been proved clinically and in animal researches in China [177-181].

Liu et al. [182] studied the effect of aqueous extract $(8 \mathrm{~g} / \mathrm{kg} /$ day $)$ to HCD-fed Wistar rats. The aqueous fraction significantly lowered TG concentration and decreased $\mathrm{TC} / \mathrm{HDL}-\mathrm{C}$ and thromboxane/prostacyclin ratio (related to platelet coagulation). The $2.5 \%$ medium-polar and nonpolar fraction mixture from aqueous fraction $(0.2 \mathrm{~g} / \mathrm{kg} / \mathrm{day}, 2$ weeks), which exhibited comparable effect on serum lipid level and stronger potency to increase the prostacyclin secretion and significantly inhibitory effect on proinflammatory interleukin 8 production, was the antiatherogenic principle of the formula. In 2013, a NMR-based metabolomics approach was conducted to elucidate the mechanisms of XFZY on HFD induced hyperlipidemia [181]. 2.5, 5, and $10 \mathrm{~g} / \mathrm{kg}$ (equivalent to crude drug mixture) of water extract of six XFZY ingredients (Bupleuri Chinensis Radix, Paeoniae Rubra Radix, Carthami Flos, Persicae Semen, Aurantii Submaturus Fructus, and Chuanxiong Rhizoma at the weight ratio of $3: 3: 3: 2: 2: 1$ ) were fed to HFD-induced SD rats for seven weeks. Statistical analysis of NMR spectra from blood plasma revealed that XFZY could attenuate hyperlipidemia, by partially reversing energy and lipid metabolism disturbance, decreasing the accumulation of $\beta$-hydroxybutyrate (ketone body) and acetyl-glycoproteins (inflammatory mediators), and enhancing glutathione biosynthesis probably due to antihyperlipidemia, antioxidative, and anti-inflammatory effects of its components.

No obvious adverse reaction was found during and after the treatment of XFZY capsules except one case reporting stomach discomfort $[167,176]$.

\section{Discussion}

TCMs have been used for over 2000 years in China. In the past few decades, they have drawn more and more attention worldwide to explore their new indications and test if they have potential to treat or prevent the disorders or dysfunctions that Western drugs or single compounds cannot easily regulate. One of the examples is hyperlipidemia. The hypolipidemic effects of TCMs have been extensively investigated and manifested in vitro and in vivo and clinically summarized in Tables 2 and 3.

In ancient TCM theory, the term "hyperlipidemia" does not exist but some scholars suggested that hyperlipidemia have many resemblances to the syndromes of dampness, turbid-phlegm, and blood stasis [183]. Taking turbid-phlegm as an example, excessive inner accumulation of phlegm in the body could block the circulation of blood and Qi, further leading to blood stasis. Patients generally have the symptoms of chest impediment, palpitations, dizziness, or even stroke in serious cases $[184,185]$. Most TCM practitioners consider the deficiency of "spleen," "kidney," or poor movement of "liver" Q $i$ as the roots (the primary aspect of a disease) of hyperlipidemia while phlegm and stasis are the tips (the secondary aspect of a disease) [53, 185].

Three of the most frequently used strategies by TCM practitioners for the prescription of TCMs [16] are (1) to relieve food retention (e.g., Oryzae cum Monasco Semen, Crataegi Fructus), enhance purgative effect (e.g., Rhei Radix et Rhizoma), and eliminate dampness and water (e.g., Alismatis Rhizoma and Ling-Gui-Zhu-Gan decoction) by targeting the gastrointestinal tract, urinary, and biliary system (e.g., Turtle jelly); (2) to promote blood circulation and relieve blood stasis by improving cardiovascular system (e.g., Salviae Miltiorrhizae Radix, Notoginseng Radix et Rhizoma, and XuefuZhuyu decoction); (3) to reinforce tonic effects by adjusting entire body functions (e.g., Ginseng Radix et Rhizoma, Danggui-Buxue decoction, and Shengmai Yin). Most of them may provide their effects via multiple approaches, such as red yeast rice that promotes blood circulation and strengthens "spleen" to improve digestions. Hence, patients with hyperlipidemia are prescribed appropriate combination of herbs according to their diagnosed syndromes so as to treat the roots well, rather than only target the tips (symptoms) only.

In addition to the long history of medical uses, in this review, preclinical researches and clinical trials provide supportive evidence to recommend the use of TCM therapy in treating hyperlipidemia.

In the Western medications, some single Western drugs clinically used alone or in coadministration with others in hyperlipidemic patients have their own adverse effects [10, $186,187]$ that may not be entirely tolerated by all patients. For example, statins use may induce incidence of myopathy in high dose or in some groups of patients [188-190]. The US National Institutes of Health suggested that many patients under statin treatment alone do not achieve the LDL-C goal [186]. As for patients who want alternative medicines for the replacement of Western medications, TCM may be their choice despite reported mild adverse side effects observed in minor cases clinically. We cannot deny that there may be similar side effects of TCM sharing similar mechanisms as conventional drugs (Figures 1-2). Red yeast rice, for instance, containing monacolins that resemble HMG-CoA reductase inhibitors may have a high potential risk. However, we do not find a report about the intake of the herb causing similar adverse effect. This is greatly attributed to the variation in the drug preparation, dosage, administration period, and population differences. In addition, Chinese herbs are seldom used alone so their synergistic effect may enhance the efficacy whereas the amount of toxic chemicals is reduced by other coexisting phytochemicals [191] in the herbal mixtures. The combination of herbs that have different pharmacological activities may have better lipid-lowering effects than their individual ingredients or even comparable to single drugs probably contributed to their actions of multiple targets. Better quality control and more efficient drug preparation may enhance TCM oral bioavailability [192] with a lower dosage used. For instance, the oral bioavailability of the Sanqi saponins in crude drug may not be satisfying whereas some adverse reactions might be caused by using the intravenous injection [102]. Using chitosan as a bioadhesive material to prepare a modified Sanqi saponins tablet improved the bioavailability after oral administration for beagle dogs [193].

More importantly, herb-drug interaction has raised growing concern to scientists despite the fact that there is growing popularity of TCM as complementary medicines with 
Western drugs in various diseases, including hyperlipidemia, CVD, and diabetes $[194,195]$. The literature also warns that there are potentially serious adverse effects and interactions between conventional therapies and TCMs $[195,196]$. We found that adverse interactions exist in the conventional drugs with TCMs in the hyperlipidemic treatment while the synergistic effect of hypolipidemic activities using these two types of medicines simultaneously is also possible. For examples, the common hypolipidemic red rice yeast is likely to have drug interactions with niacin that has long been used to increase blood HDL levels. This is because the herb contains monacolins that resemble the pharmacokinetics of lovastatin which has synergistic effect with niacin. Possible adverse effects include flushing, pruritus, rash, or gastrointestinal adverse events [197]. In particular, hyperlipidemic patients who are generally suffering from CVD and taking conventional anticoagulants and antiplatelet drugs should not ignore the potential risks with TCMs [93]. Warfarin, an anticoagulant, is often found to interact with TCMs such as Salviae Miltiorrhizae Radix [136, 137] and Ginseng Radix et Rhizoma [92], increasing the bleeding risk of the patients. The TCM formulation composed of these herbs such as Shengmai Yin [170] and Danshen-Gegen formula [150] should also avoid coadministration of the anticoagulant.

In the contrast, the combination of TCM bioactive compounds and Western drugs may give better hypolipidemic activities than using them solely, such as the fermented red ginseng powder plus levan [88] and berberine from Coptidis Rhizoma with simvastatin [68]. This may reduce the dose of the single drugs supplemented with TCM products for those who do not tolerate these drugs well.

Since there are insufficient information about the effects of interactions of TCMs with the conventional hypolipidemic medications provided to the clinics and the general public, patients may underestimate or even may not be aware of the potential danger of herb-drug interactions which are similar to drug-drug interactions in terms of their effects on ADME properties [194, 198]. More investigation of herb-drug interactions in vivo and clinically with the main phytochemicals from the single Chinese herbs and more complicated TCM formulae is of high importance to compensate our limited knowledge of TCMs. Since little is known, Chinese medicine practitioners and clinicians should warn hyperlipidemic patients about the potential risks of herb-drug interactions of TCMs with Western drugs, particularly those taking anticoagulants and antiplatelet drugs.

Inconsistencies in the hypolipidemic effect of the herb were observed in different studies of this review. It may be attributed to the composition of TCM extract used in the experiments, administration method, dosages, animal models, and duration. Also, due to the limitation of present scientific technology, biological mechanisms of many other TCM formulae have not been completely elucidated [199]. More systematic, well-designed animal and randomized clinical studies in chronic administration with sufficient sample sizes are essential to investigate their exact mechanisms of the hypolipidemic effects, safety, and pharmacokinetics so as to give a more effective alternative to the hyperlipidemic patients.

\section{Conclusion}

Most of single herbs and formulae demonstrated the improvement of hyperlipidemic conditions with multiple and diverse mechanisms of actions in spite of their mild side effects. As more and more people tend to use TCM as an alternative medicine, more extensive, well-designed preclinical and clinical trials on the potential synergistic and adverse side effects of herb-drug interactions, as well as their mechanisms, are warranted. Hyperlipidemic patients should be warned about the potential risks of herb-drug interactions, particularly those taking anticoagulants and antiplatelet drugs.

\section{Abbreviations}

ACAT: Acyl CoA-cholesterol acyl-transferase

ACSL: Long-chain acyl-CoA synthetase

Apo: Apolipoprotein

CE: $\quad$ Cholesterol esters

CVD: $\quad$ Cardiovascular diseases

CP: $\quad$ Pharmacopoeia of the People's Republic of

CPT1A: $\quad$ Carnitine palmitoyltransferase-1A

FA: $\quad$ Fatty acids

FXR: $\quad$ Farnesoid X receptor

LXR: $\quad$ Liver X receptor

HCD: High-cholesterol diet

HDL-C: High density lipoprotein cholesterol,

HFD: High-fat diet

HMG-CoA: 3-Hydroxy-3-methyl-glutaryl-coenzyme

$$
\text { A }
$$

ICAM-1: Intercellular adhesion molecule-1

LDL-C: Low-density lipoprotein cholesterol

LDLR: LDL receptors

LPL: $\quad$ Lipoprotein lipase

MCP-1: Monocyte chemo-attractant protein-1

MDA: Malondialdehyde

NAFLD: Nonalcoholic fatty liver disease

NO: $\quad$ Nitric oxide

OVX: Ovariectomized

Ox-LDL: Oxidized LDL

PEPCK: Phosphoenolpyruvate carboxykinase

PPAR: $\quad$ Peroxisome proliferator-activated

$\begin{array}{ll} & \text { receptors } \\ \text { ROS: } & \text { Reactive oxygen species }\end{array}$

SD: $\quad$ Sprague-Dawley

SOD: $\quad$ Superoxide dismutase

SREBP-1c: Sterol regulatory element-binding protein $1 \mathrm{c}$

TC: $\quad$ Total cholesterols

TCM: Traditional Chinese medicine

TG: $\quad$ Triglycerides

TR $\beta$ 1: $\quad$ Thyroid hormone receptor $\beta 1$

VLDL: Very low-density lipoproteins. 


\section{Conflict of Interests}

The authors declare that there is no conflict of interests regarding the publication of this paper.

\section{Acknowledgments}

The study was supported by the Shenzhen Municipal Key Laboratory Advancement Program, Shenzhen, China (Project nos. CXB201104220020A and ZDSY20120618173912712). The authors thank Mr. TzeTim Lee and Ms. Rosalynn Tsun-Ling Ng for proofreading the paper.

\section{References}

[1] V. G. Athyros, K. Tziomalos, A. Karagiannis, and D. P. Mikhailidis, "Dyslipidaemia of obesity, metabolic syndrome and type 2 diabetes mellitus: the case for residual risk reduction after statin treatment," The Open Cardiovascular Medicine Journal, vol. 5, pp. 24-34, 2011.

[2] P. K. Shah, S. Kaul, J. Nilsson, and B. Cercek, "Exploiting the vascular protective effects of high-density lipoprotein and its apolipoproteins: an idea whose time for testing is coming, part I," Circulation, vol. 104, no. 19, pp. 2376-2383, 2001.

[3] P. Durrington, “Dyslipidaemia," The Lancet, vol. 362, no. 9385, pp. 717-731, 2003.

[4] A. S. Go, D. Mozaffarian, V. L. Roger et al., "Heart disease and stroke statistics-2013 update: a Report from the American Heart Association," Circulation, vol. 127, no. 1, p. e6-e245, 2013.

[5] D. M. Kusters, H. J. Avis, M. J. Braamskamp et al., "Inheritance pattern of familial hypercholesterolemia and markers of cardiovascular risk," Journal of Lipid Research, vol. 54, no. 9, pp. 25432549, 2013.

[6] P. J. Talmud, S. Shah, R. Whittall et al., "Use of low-density lipoprotein cholesterol gene score to distinguish patients with polygenic and monogenic familial hypercholesterolaemia: a case-control study," The Lancet, vol. 381, no. 9874, pp. 1293-1301, 2013.

[7] J. L. Goldstein, H. G. Schrott, W. R. Hazzard, E. L. Bierman, and A. G. Motulsky, "Hyperlipidemia in coronary heart disease. II. Genetic analysis of lipid levels in 176 families and delineation of a new inherited disorder, combined hyperlipidemia," The Journal of Clinical Investigation, vol. 52, no. 7, pp. 1544-1568, 1973.

[8] D. S. Fredrickson, R. I. Levy, and R. S. Lees, "Fat transport in lipoproteins - an integrated approach to mechanisms and disorders," The New England Journal of Medicine, vol. 276, no. 1, pp. 34-42, 1967.

[9] T. T. Hlaing and A. Park, "Hyperlipidaemia," Medicine, vol. 41, no. 10, pp. 607-609, 2013.

[10] S. P. Mahamuni, R. D. Khose, F. Menaa, and S. L. Badole, "Therapeutic approaches to drug targets in hyperlipidemia," BioMedicine, vol. 2, no. 4, pp. 137-146, 2012.

[11] P. R. Deepa and P. Varalakshmi, "Atheroprotective effect of exogenous heparin-derivative treatment on the aortic disturbances and lipoprotein oxidation in hypercholesterolemic diet fed rats," Clinica Chimica Acta, vol. 355, no. 1-2, pp. 119-130, 2005.
[12] C. D. Mathers and D. Loncar, "Projections of global mortality and burden of disease from 2002 to 2030," PLoS Medicine, vol. 3, no. 11, article e442, 2006.

[13] G. A. Roth, S. D. Fihn, A. H. Mokdad, W. Aekplakorn, T. Hasegawa, and S. S. Lim, "High total serum cholesterol, medication coverage and therapeutic control: an analysis of national health examination survey data from eight countries," Bulletin of the World Health Organization, vol. 89, no. 2, pp. 92101, 2011.

[14] P. P. Toth, "Drug treatment of hyperlipidaemia: a guide to the rational use of lipid-lowering drugs," Drugs, vol. 70, no. 11, pp. 1363-1379, 2010.

[15] A. M. Gotto, "Cholesterol management in theory and practice," Circulation, vol. 96, no. 12, pp. 4424-4430, 1997.

[16] W. Xie, Y. Zhao, and L. Du, "Emerging approaches of traditional Chinese medicine formulas for the treatment of hyperlipidemia," Journal of Ethnopharmacology, vol. 140, no. 2, pp. 345367, 2012.

[17] M. M. Hussain, "Intestinal lipid absorption and lipoprotein formation," Current Opinion in Lipidology, vol. 25, no. 3, pp. 200-206, 2014.

[18] A. J. Lusis and P. Pajukanta, "A treasure trove for lipoprotein biology," Nature Genetics, vol. 40, no. 2, pp. 129-130, 2008.

[19] Y. Stein and O. Stein, "The Origin and Fate of LDL," in Atherosclerosis, G. Manning and M. D. Haust, Eds., pp. 413-420, Springer, New York, NY, USA, 1977.

[20] C. J. Fielding and P. E. Fielding, "Molecular physiology of reverse cholesterol transport," Journal of Lipid Research, vol. 36, no. 2, pp. 211-228, 1995.

[21] C. Verdier, L. O. Martinez, J. Ferrières et al., "Targeting highdensity lipoproteins: update on a promising therapy," Archives of Cardiovascular Diseases, vol. 106, no. 11, pp. 601-611, 2013.

[22] N. Y. Kalaany and D. J. Mangelsdorf, "LXRs and FXR: the Yin and Yang of cholesterol and fat metabolism," Annual Review of Physiology, vol. 68, no. 1, pp. 159-191, 2006.

[23] S. Deakin, I. Leviev, M. Gomaraschi, L. Calabresi, G. Franceschini, and R. W. James, "Enzymatically active paraoxonase-1 is located at the external membrane of producing cells and released by a high affinity, saturable, desorption mechanism," The Journal of Biological Chemistry, vol. 277, no. 6, pp. 43014308, 2002.

[24] A. Kontush, S. Chantepie, and M. J. Chapman, "Small, dense HDL particles exert potent protection of atherogenic LDL against oxidative stress," Arteriosclerosis, Thrombosis, and Vascular Biology, vol. 23, no. 10, pp. 1881-1888, 2003.

[25] C. Mineo, H. Deguchi, J. H. Griffin, and P. W. Shaul, "Endothelial and antithrombotic actions of HDL," Circulation Research, vol. 98, no. 11, pp. 1352-1364, 2006.

[26] B. J. Ansell, M. Navab, S. Hama et al., "Inflammatory/ antiinflammatory properties of high-density lipoprotein distinguish patients from control subjects better than high-density lipoprotein cholesterol levels and are favorably affected by simvastatin treatment," Circulation, vol. 108, no. 22, pp. 27512756, 2003.

[27] J. Bernard, "Free fatty acid receptor family: novel targets for the treatment of diabetes and dyslipidemia," Current Opinion in Investigational Drugs, vol. 9, no. 10, pp. 1078-1083, 2008.

[28] M. A. Micallef and M. L. Garg, "Beyond blood lipids: phytosterols, statins and omega-3 polyunsaturated fatty acid therapy for hyperlipidemia," The Journal of Nutritional Biochemistry, vol. 20, no. 12, pp. 927-939, 2009. 
[29] T. Funatsu, K. Suzuki, M. Goto et al., "Prolonged inhibition of cholesterol synthesis by atorvastatin inhibits apo B-100 and triglyceride secretion from HepG2 cells," Atherosclerosis, vol. 157, no. 1, pp. 107-115, 2001.

[30] P. O. Kwiterovich Jr., "Clinical relevance of the biochemical, metabolic, and genetic factors that influence low-density lipoprotein heterogeneity," American Journal of Cardiology, vol. 90, no. 8, pp. 30-47, 2002.

[31] L. Mabile, O. Meilhac, I. Escargueil-Blanc et al., "Mitochondrial function is involved in LDL oxidation mediated by human cultured endothelial cells," Arteriosclerosis, Thrombosis, and Vascular Biology, vol. 17, no. 8, pp. 1575-1582, 1997.

[32] M. K. Cathcart, D. W. Morel, and G. M. Chisolm III, "Monocytes and neutrophils oxidize low density lipoprotein making it cytotoxic," Journal of Leukocyte Biology, vol. 38, no. 2, pp. 341350, 1985.

[33] R. Ross, "The pathogenesis of atherosclerosis: a perspective for the 1990s," Nature, vol. 362, no. 6423, pp. 801-809, 1993.

[34] A. Blanco-Molina, D. Martín-Escalante, D. Bravo et al., "Highdensity lipoproteins protect endothelial cells from apoptosis induced by oxidized low-density lipoproteins," Protoplasma, vol. 211, no. 3-4, pp. 198-206, 2000.

[35] M. Jacobs, F. Plane, and K. R. Bruckdorfer, "Native and oxidized low-density lipoproteins have different inhibitory effects on endothelium-derived relaxing factor in the rabbit aorta," British Journal of Pharmacology, vol. 100, no. 1, pp. 21-26, 1990.

[36] H. Chan, M. Lougheed, I. Laher, and U. P. Steinbrecher, "Oxidized low-density lipoprotein inhibits endotheliumdependent vasodilation by an antioxidant-sensitive, lysophosphatidylcholine-independent mechanism," Journal of Cardiovascular Pharmacology, vol. 41, no. 6, pp. 856-865, 2003.

[37] C. P. Sparrow, S. Parthasarathy, and D. Steinberg, "A macrophage receptor that recognizes oxidized low density lipoprotein but not acetylated low density lipoprotein," The Journal of Biological Chemistry, vol. 264, no. 5, pp. 2599-2604, 1989.

[38] T. Gordon and W. B. Kannel, "Premature mortality from coronary heart disease. The Framingham study," The Journal of the American Medical Association, vol. 215, no. 10, pp. 1617-1625, 1971.

[39] P. Holvoet, N. S. Jenny, P. J. Schreiner, R. P. Tracy, and D. R. Jacobs, "The relationship between oxidized LDL and other cardiovascular risk factors and subclinical CVD in different ethnic groups: the Multi-Ethnic Study of Atherosclerosis (MESA)," Atherosclerosis, vol. 194, no. 1, pp. 245-252, 2007.

[40] J. D. Brown and J. Plutzky, "Peroxisome proliferator-activated receptors as transcriptional nodal points and therapeutic targets," Circulation, vol. 115, no. 4, pp. 518-533, 2007.

[41] B. Staels, J. Dallongeville, J. Auwerx, K. Schoonjans, E. Leitersdorf, and J.-C. Fruchart, "Mechanism of action of fibrates on lipid and lipoprotein metabolism," Circulation, vol. 98, no. 19, pp. 2088-2093, 1998.

[42] T. Sudhop, D. Lütjohann, A. Kodal et al., "Inhibition of intestinal cholesterol absorption by ezetimibe in humans," Circulation, vol. 106, no. 15, pp. 1943-1948, 2002.

[43] M. H. Davidson, "A systematic review of bile acid sequestrant therapy in children with familial hypercholesterolemia," Journal of Clinical Lipidology, vol. 5, no. 2, pp. 76-81, 2011.

[44] G. L. Vega, N. B. Cater, S. Meguro, and S. M. Grundy, "Influence of extended-release nicotinic acid on nonesterified fatty acid flux in the metabolic syndrome with atherogenic dyslipidemia,"
American Journal of Cardiology, vol. 95, no. 11, pp. 1309-1313, 2005.

[45] C. S. Vaccari, R. A. Hammoud, S. H. Nagamia, K. Ramasamy, A. L. Dollar, and B. V. Khan, "Revisiting niacin: reviewing the evidence," Journal of Clinical Lipidology, vol. 1, no. 4, pp. 248255, 2007.

[46] A. C. Rustan, J. O. Nossen, E. N. Christiansen, and C. A. Drevon, "Eicosapentaenoic acid reduces hepatic synthesis and secretion of triacylglycerol by decreasing the activity of acyl-coenzyme A:1,2-diacylglycerol acyltransferase," Journal of Lipid Research, vol. 29, no. 11, pp. 1417-1426, 1988.

[47] J. S. Parks, F. L. Johnson, M. D. Wilson, and L. L. Rudel, "Effect of fish oil diet on hepatic lipid metabolism in nonhuman primates: lowering of secretion of hepatic triglyceride but not apoB," Journal of Lipid Research, vol. 31, no. 3, pp. 455-466, 1990.

[48] W. S. Harris, M. Miller, A. P. Tighe, M. H. Davidson, and E. J. Schaefer, "Omega-3 fatty acids and coronary heart disease risk: clinical and mechanistic perspectives," Atherosclerosis, vol. 197, no. 1, pp. 12-24, 2008.

[49] R. Hou and A. C. Goldberg, "Lowering low-density lipoprotein cholesterol: statins, ezetimibe, bile acid sequestrants, and combinations: comparative efficacy and safety," Endocrinology and Metabolism Clinics of North America, vol. 38, no. 1, pp. 79-97, 2009.

[50] P. Kardas, "Prevalence and reasons for non-adherence to hyperlipidemia treatment," Central European Journal of Medicine, vol. 8, no. 5, pp. 539-547, 2013.

[51] P. H. Langsjoen, J. O. Langsjoen, A. M. Langsjoen, and L. A. Lucas, "Treatment of statin adverse effects with supplemental Coenzyme Q 10 and statin drug discontinuation," BioFactors, vol. 25, no. 1-4, pp. 147-152, 2005.

[52] D. Rozman and K. Monostory, "Perspectives of the non-statin hypolipidemic agents," Pharmacology and Therapeutics, vol. 127, no. 1, pp. 19-40, 2010.

[53] X. B. Dou, X. D. Wo, and C. L. Fan, "Progress of research in treatment of hyperlipidemia by monomer or compound recipe of Chinese herbal medicine," Chinese Journal of Integrative Medicine, vol. 14, no. 1, pp. 71-75, 2008.

[54] Chinese Pharmacopoeia Commission, Pharmacopoeia of the People's Republic of China, Chinese Medical Science Press, Beijing, China, 2010.

[55] W. B. Qian, H. Pang, D. Q. Xie, L. Y. Xi, and H. Y. Zhang, "Preparation and research into the effects on the decrease of the hyperlipidemica of mice of water and alcohol extracts of Alisma orientalis," Journal of Mathematical Medicine, vol. 20, no. 6, pp. 836-838, 2007.

[56] X. Y. Yu, J. H. Zhong, and X. Zhang, "Hypolipidemic effects and its chemical principles of Ze Xie," Chinese Medicine Modern Distance Education of China, vol. 8, no. 11, p. 250, 2010.

[57] H. Dan, J. Wu, M. Peng et al., "Hypolipidemic effects of Alismatis Rhizome on lipid profile in mice fed high-fat diet," Saudi Medical Journal, vol. 32, no. 7, pp. 701-707, 2011.

[58] X. Hong, H. Tang, L. Wu, and A. Li, "Protective effects of the Alisma orientalis extract on the experimental nonalcoholic fatty liver disease," Journal of Pharmacy and Pharmacology, vol. 58, no. 10, pp. 1391-1398, 2006.

[59] Y. Imai, H. Matsumura, and Y. Aramaki, "Hypocholesterolemic effect of alisol A-24-monoacetate and its related compounds in rats," The Japanese Journal of Pharmacology, vol. 20, no. 2, pp. $222-228,1970$. 
[60] M. Q. Huang, W. Xu, S. S. Wu, J. J. Lu, and X. P. Chen, "A 90-day subchronic oral toxicity study of triterpene-enriched extract from Alismatis Rhizoma in rats," Food and Chemical Toxicology, vol. 58, pp. 318-323, 2013.

[61] M. F. Yuen, S. Tam, J. Fung, D. K. H. Wong, B. C. Y. Wong, and C. L. Lai, "Traditional Chinese medicine causing hepatotoxicity in patients with chronic hepatitis B infection: a 1-year prospective study," Alimentary Pharmacology and Therapeutics, vol. 24, no. 8, pp. 1179-1186, 2006.

[62] X. Zhao, L. Lu, Y. Zhang, and B. Zhang, "Study on discriminating nephrotoxic components in Zexie," China Journal of Chinese Materia Medica, vol. 36, no. 6, pp. 758-761, 2011.

[63] T. Yokozawa, A. Ishida, E. J. Cho, and T. Nakagawa, "The effects of Coptidis Rhizoma extract on a hypercholesterolemic animal model," Phytomedicine, vol. 10, no. 1, pp. 17-22, 2003.

[64] Y. Cao, W. Bei, Y. Hu et al., "Hypocholesterolemia of Rhizoma Coptidis alkaloids is related to the bile acid by up-regulated CYP7A1 in hyperlipidemic rats," Phytomedicine, vol. 19, no. 89, pp. 686-692, 2012.

[65] W. Xie, D. Gu, J. Li, K. Cui, and Y. Zhang, "Effects and action mechanisms of berberine and Rhizoma Coptidis on gut microbes and obesity in high-fat diet-fed C57BL/6J mice," PLoS ONE, vol. 6, no. 9, Article ID e24520, 2011.

[66] W. Kong, J. Wei, P. Abidi et al., "Berberine is a novel cholesterollowering drug working through a unique mechanism distinct from statins," Nature Medicine, vol. 10, no. 12, pp. 1344-1351, 2004.

[67] Y. Hu, E. A. Ehli, J. Kittelsrud et al., "Lipid-lowering effect of berberine in human subjects and rats," Phytomedicine, vol. 19, no. 10, pp. 861-867, 2012.

[68] W. J. Kong, J. Wei, Z. Y. Zuo et al., "Combination of simvastatin with berberine improves the lipid-lowering efficacy," Metabolism: Clinical and Experimental, vol. 57, no. 8, pp. 10291037, 2008.

[69] D. H. Kuo, C. H. Yeh, P. C. Shieh, K. C. Cheng, F. A. Chen, and J. T. Cheng, "Effect of ShanZha, a Chinese herbal product, on obesity and dyslipidemia in hamsters receiving high-fat diet," Journal of Ethnopharmacology, vol. 124, no. 3, pp. 544-550, 2009.

[70] J. Y. Zhang, R. X. Liang, L. Wang et al., "Effects of an aqueous extract of Crataegus pinnatifida Bge. var. major NEBr. fruit on experimental atherosclerosis in rats," Journal of Ethnopharmacology, vol. 148, no. 2, pp. 563-569, 2013.

[71] Y. Lin, M. A. Vermeer, and E. A. Trautwein, "Triterpenic acids present in hawthorn lower plasma cholesterol by inhibiting intestinal ACAT activity in hamsters," Evidence-Based Complementary and Alternative Medicine, vol. 2011, Article ID 801272, 9 pages, 2011.

[72] C. Y. Kwok, C. N. Y. Wong, M. Y. C. Yau et al., "Consumption of dried fruit of Crataegus pinnatifida (hawthorn) suppresses highcholesterol diet-induced hypercholesterolemia in rats," Journal of Functional Foods, vol. 2, no. 3, pp. 179-186, 2010.

[73] M. H. Pittler, K. Schmidt, and E. Ernst, "Hawthorn extract for treating chronic heart failure: meta-analysis of randomized trials," The American Journal of Medicine, vol. 114, no. 8, pp. 665674, 2003.

[74] M. H. Pittler, R. Guo, and E. Ernst, "Hawthorn extract for treating chronic heart failure," Cochrane Database of Systematic Reviews, no. 1, p. CD005312, 2008.

[75] C. Daniele, G. Mazzanti, M. H. Pittler, and E. Ernst, "Adverseevent profile of Crataegus spp.: a systematic review," Drug Safety, vol. 29, no. 6, pp. 523-535, 2006.
[76] S. H. Kim and K. S. Park, "Effects of Panax ginseng extract on lipid metabolism in humans," Pharmacological Research, vol. 48, no. 5, pp. 511-513, 2003.

[77] D. H. M. El-Farok and E. S. El-Denshry, "Lipid lowering effect of ginseng and alpha-lipoic acid in hypercholesterolemic patients," Global Journal of Pharmacology, vol. 7, no. 3, pp. 298-306, 2013.

[78] M. Inoue, C. Z. Wu, D. Q. Dou, Y. J. Chen, and Y. Ogihara, "Lipoprotein lipase activation by red Ginseng saponins in hyperlipidemia model animals," Phytomedicine, vol. 6, no. 4, pp. 257-265, 1999.

[79] S. Y. Hwang, D. J. Son, I. W. Kim et al., "Korean red ginseng attenuates hypercholesterolemia-enhanced platelet aggregation through suppression of diacylglycerol liberation in highcholesterol-diet-fed rabbits," Phytotherapy Research, vol. 22, no. 6, pp. 778-783, 2008.

[80] S. Lim, J. W. Yoon, S. H. Choi et al., "Effect of ginsam, a vinegar extract from Panax ginseng, on body weight and glucose homeostasis in an obese insulin-resistant rat model," Metabolism: Clinical and Experimental, vol. 58, no. 1, pp. 8-15, 2009.

[81] Y. S. Kwak, J. S. Kyung, J. S. Kim, J. Y. Cho, and M. H. Rhee, "Anti-hyperlipidemic effects of red ginseng acidic polysaccharide from Korean red ginseng," Biological and Pharmaceutical Bulletin, vol. 33, no. 3, pp. 468-472, 2010.

[82] N. Karu, R. Reifen, and Z. Kerem, "Weight gain reduction in mice fed Panax ginseng saponin, a pancreatic lipase inhibitor," Journal of Agricultural and Food Chemistry, vol. 55, no. 8, pp. 2824-2828, 2007.

[83] L. S. Lee, C. W. Cho, H. D. Hong et al., "Hypolipidemic and antioxidant properties of phenolic compound-rich extracts from white ginseng (Panax ginseng) in cholesterol-fed rabbits," Molecules, vol. 18, no. 10, pp. 12548-12560, 2013.

[84] H. L. Jung and H. Y. Kang, "Effects of Korean red ginseng supplementation on muscle glucose uptake in high-fat fed rats," Chinese Journal of Natural Medicines, vol. 11, no. 5, pp. 494-499, 2013.

[85] N. Lin, D. L. Cai, D. Jin, Y. Chen, and J. J. Shi, "Ginseng panaxoside $\mathrm{Rbl}$ reduces body weight in diet-induced obese mice," Cell Biochemistry and Biophysics, vol. 68, no. 1, pp. 189194, 2014.

[86] S. Lee, M. S. Lee, C. T. Kim, I. H. Kim, and Y. Kim, "Ginsenoside RG3 reduces lipid accumulation with AMP-activated protein kinase (AMPK) activation in HepG2 cells," International Journal of Molecular Sciences, vol. 13, no. 5, pp. 5729-5739, 2012.

[87] H. G. Kim, S. R. Yoo, H. J. Park et al., "Antioxidant effects of Panax ginseng C.A. Meyer in healthy subjects: a randomized, placebo-controlled clinical trial," Food and Chemical Toxicology, vol. 49, no. 9, pp. 2229-2235, 2011.

[88] J. S. Oh, S. R. Lee, K. T. Hwang, and G. E. Ji, “The anti-obesity effects of the dietary combination of fermented red ginseng with levan in high fat diet mouse model," Phytotherapy Research, vol. 28, no. 4, pp. 617-622, 2014.

[89] N. H. Lee and C. G. Son, "Systematic review of randomized controlled trials evaluating the efficacy and safety of ginseng," Journal of Acupuncture and Meridian Studies, vol. 4, no. 2, pp. 85-97, 2011.

[90] R. K. Siegel, "Ginseng abuse syndrome. Problems with the panacea," Journal of the American Medical Association, vol. 241, no. 15, pp. 1614-1615, 1979.

[91] B. D. Jones and A. M. Runikis, "Interaction of ginseng with phenelzine," Journal of Clinical Psychopharmacology, vol. 7, no. 3, pp. 201-202, 1987. 
[92] S. H. Lee, Y. M. Ahn, S. Y. Ahn, H. K. Doo, and B. C. Lee, "Interaction between warfarin and Panax ginseng in ischemic stroke patients," Journal of Alternative and Complementary Medicine, vol. 14, no. 6, pp. 715-721, 2008.

[93] H. H. Tsai, H. W. Lin, Y. H. Lu, Y. L. Chen, and G. B. Mahady, "A review of potential harmful interactions between anticoagulant/antiplatelet agents and Chinese herbal medicines," PLoS ONE, vol. 8, no. 5, Article ID e64255, 2013.

[94] State Administration of Traditional Chinese Medicine, Chinese Materia Medica (Zhonghua Bencao in Chinese), Shanghai Scientific and Technical Publishers, Shanghai, China, 1998.

[95] W. Xia, C. Sun, Y. Zhao, and L. Wu, "Hypolipidemic and antioxidant activities of Sanchi (Radix Notoginseng) in rats fed with a high fat diet," Phytomedicine, vol. 18, no. 6, pp. 516-520, 2011.

[96] J. H. Wu, G. P. H. Leung, Y. W. Kwan et al., "Suppression of diet-induced hypercholesterolaemia by saponins from Panax notoginseng in rats," Journal of Functional Foods, vol. 5, no. 3, pp. 1159-1169, 2013.

[97] Y. G. Zhang, H. G. Zhang, G. Y. Zhang et al., "Panax notoginseng saponins attenuate atherosclerosis in rats by regulating the blood lipid profile and an anti-inflammatory action," Clinical and Experimental Pharmacology and Physiology, vol. 35, no. 10, pp. 1238-1244, 2008.

[98] W. Ji and B. Q. Gong, "Hypolipidemic effects and mechanisms of Panax notoginseng on lipid profile in hyperlipidemic rats," Journal of Ethnopharmacology, vol. 113, no. 2, pp. 318-324, 2007.

[99] A. F. G. Cicero, G. Vitale, G. Savino, and R. Arletti, "Panax notoginseng (Burk.) Effects on fibrinogen and lipid plasma level in rats fed on a high-fat diet," Phytotherapy Research, vol. 17, no. 2, pp. 174-178, 2003.

[100] H. Sinzinger and M. Rodrigues, "Atorvastatin and fibrinogena small subgroup shows extreme response," Atherosclerosis, vol. 145, no. 2, pp. 415-417, 1999.

[101] A. S. Wierzbicki, P. J. Lumb, Y. K. Semra, and M. A. Crook, "Effect of atorvastatin on plasma fibrinogen," The Lancet, vol. 351, no. 9102, pp. 569-570, 1998.

[102] Y. Chen, Y. Li, and G. Zhu, "Analysis of 103 cases with adverse drug reactions induced by sanqizongzaogan injection," China Journal of Chinese Materia Medica, vol. 35, no. 2, pp. 237-239, 2010.

[103] G. Wang, L. Wang, Z. Y. Xiong, B. Mao, and T. Q. Li, "Compound salvia pellet, a traditional Chinese medicine, for the treatment of chronic stable angina pectoris compared with nitrates: a meta-analysis," Medical Science Monitor, vol. 12, no. 1, pp. SR1-SR7, 2006.

[104] J. Ma, Y. Li, Q. Ye et al., "Constituents of red yeast rice, a traditional Chinese food and medicine," Journal of Agricultural and Food Chemistry, vol. 48, no. 11, pp. 5220-5225, 2000.

[105] L. Childress, A. Gay, A. Zargar, and M. K. Ito, "Review of red yeast rice content and current Food and Drug Administration oversight," Journal of Clinical Lipidology, vol. 7, no. 2, pp. 117-122, 2013.

[106] M. Journoud and P. J. H. Jones, "Red yeast rice: a new hypolipidemic drug," Life Sciences, vol. 74, no. 22, pp. 26752683, 2004

[107] O. Gheith, H. Sheashaa, M. Abdelsalam, Z. Shoeir, and M. Sobh, "Efficacy and safety of Monascus purpureus Went rice in children and young adults with secondary hyperlipidemia: a preliminary report," European Journal of Internal Medicine, vol. 20, no. 3, pp. e57-e61, 2009.
[108] C. W. Yang and S. A. Mousa, "The effect of red yeast rice (Monascus purpureus) in dyslipidemia and other disorders," Complementary Therapies in Medicine, vol. 20, no. 6, pp. 466474, 2012.

[109] W. Wei, C. Li, Y. Wang, H. Su, J. Zhu, and D. Kritchevsky, "Hypolipidemic and anti-atherogenic effects of long-term Cholestin (Monascus purpureus-fermented rice, red yeast rice) in cholesterol fed rabbits," The Journal of Nutritional Biochemistry, vol. 14, no. 6, pp. 314-318, 2003.

[110] M. P. Bogsrud, L. Ose, G. Langslet et al., "HypoCol (red yeast rice) lowers plasma cholesterol-a randomized placebo controlled study," Scandinavian Cardiovascular Journal, vol. 44, no. 4, pp. 197-200, 2010.

[111] J. Liu, J. Zhang, Y. Shi, S. Grimsgaard, T. Alraek, and V. Fønnebø, "Chinese red yeast rice (Monascus purpureus) for primary hyperlipidemia: a meta-analysis of randomized controlled trials," Chinese Medicine, vol. 1, article 4, 2006.

[112] C. D. Liao, Y. C. Chen, H. Y. Lin, L. C. Chiueh, and D. Y. C. Shih, "Incidence of citrinin in red yeast rice and various commercial Monascus products in Taiwan from 2009 to 2012," Food Control, vol. 38, pp. 178-183, 2014.

[113] L. P. Yan, S. W. Chan, A. S. C. Chan, S. L. Chen, X. J. Ma, and H. $\mathrm{X} . \mathrm{Xu}$, "Puerarin decreases serum total cholesterol and enhances thoracic aorta endothelial nitric oxide synthase expression in diet-induced hypercholesterolemic rats," Life Sciences, vol. 79, no. 4, pp. 324-330, 2006.

[114] D. W. Lim, J. G. Kim, and Y. T. Kim, "Effects of dietary isoflavones from Puerariae Radix on lipid and bone metabolism in ovariectomized rats," Nutrients, vol. 5, no. 7, pp. 2734-2746, 2013.

[115] N. Peng, J. K. Prasain, Y. Dai et al., "Chronic dietary kudzu isoflavones improve components of metabolic syndrome in stroke-prone spontaneously hypertensive rats," Journal of Agricultural and Food Chemistry, vol. 57, no. 16, pp. 7268-7273, 2009.

[116] J. F. Wang, Y. X. Guo, J. Z. Niu, J. Liu, L. Q. Wang, and P. H. Li, "Effects of Radix Puerariae flavones on liver lipid metabolism in ovariectomized rats," World Journal of Gastroenterology, vol. 10, no. 13, pp. 1967-1970, 2004.

[117] C. T. Wu, J. N. Tzeng, J. N. Lai, S. H. Tsan, and J. D. Wang, "Prescription profile of Chinese herbal products containing coumestrol, genestein, and/or daidzein among female users: an analysis of national health insurance data in Taiwan between 1997 and 2007," Chinese Medicine, vol. 7, article 22, 2012.

[118] H. M. Chiang, S. H. Fang, K. C. Wen et al., "Life-threatening interaction between the root extract of Pueraria lobata and methotrexate in rats," Toxicology and Applied Pharmacology, vol. 209, no. 3, pp. 263-268, 2005.

[119] Japanese Pharmacopoeia Committee, The Japanese Pharmacopoeia, Society of Japanese Pharmacopoeia, Tokyo, Japan, 15th edition, 2006.

[120] British Pharmacopoeia Commission, British Pharmacopoeia 2009, The Stationery Office on Behalf of the Medicines and Healthcare Products Regulatory Agency, London, UK, 2008.

[121] European Pharmacopoeia Commission, European Pharmacopoeia, European Directorate for the Quality of Medicines and Healthcare of Council of Europe, Strasbourg, France, 7th edition, 2010.

[122] The United States Pharmacopeial Convention, The United States Pharmacopeia/ National Formulary, The United States Pharmacopeial Convention, Rochville, Md, USA, 2009. 
[123] Q. Gao, W. S. Qin, Z. H. Jia et al., "Rhein improves renal lesion and ameliorates dyslipidemia in $\mathrm{db} / \mathrm{db}$ mice with diabetic nephropathy," Planta Medica, vol. 76, no. 1, pp. 27-33, 2010.

[124] X. Sheng, M. Wang, M. Lu, B. Xi, H. Sheng, and Y. Q. Zang, "Rhein ameliorates fatty liver disease through negative energy balance, hepatic lipogenic regulation, and immunomodulation in diet-induced obese mice," American Journal of Physiology: Endocrinology and Metabolism, vol. 300, no. 5, pp. E886-E893, 2011.

[125] X. Sheng, X. Zhu, Y. Zhang et al., "Rhein protects against obesity and related metabolic disorders through liver $\mathrm{X}$ receptormediated uncoupling protein 1 upregulation in brown adipose tissue," International Journal of Biological Sciences, vol. 8, no. 10, pp. 1375-1384, 2012.

[126] Y. F. Liu, H. M. Yu, C. Zhang et al., "Treatment with rhubarb improves brachial artery endothelial function in patients with atherosclerosis: a randomized, double-blind, placebocontrolled clinical trial," American Journal of Chinese Medicine, vol. 35, no. 4, pp. 583-595, 2007.

[127] Y. Liu, F. Yan, Y. Liu et al., "Aqueous extract of rhubarb stabilizes vulnerable atherosclerotic plaques due to depression of inflammation and lipid accumulation," Phytotherapy Research, vol. 22, no. 7, pp. 935-942, 2008.

[128] Y. Qin, J. B. Wang, W. J. Kong et al., "The diarrhoeogenic and antidiarrhoeal bidirectional effects of rhubarb and its potential mechanism," Journal of Ethnopharmacology, vol. 133, no. 3, pp. 1096-1102, 2011.

[129] W. H. Organisation, WHO Monographs on Selected Medicinal Plants, World Health Organisation, Geneva, Switzerland, 1999.

[130] C. M. Li, X. L. Dong, X. D. Fan et al., "Aqueous extract of danshen (Salvia miltiorrhiza Bunge) protects ovariectomized rats fed with high-fat diet from endothelial dysfunction," Menopause, vol. 20, no. 1, pp. 100-109, 2013.

[131] S. Wassmann, A. T. Bäumer, K. Strehlow et al., "Endothelial dysfunction and oxidative stress during estrogen deficiency in spontaneously hypertensive rats," Circulation, vol. 103, no. 3, pp. 435-441, 2001.

[132] Y. L. Chen, S. P. Yang, M. S. Shiao, J. W. Chen, and S. J. Lin, "Salvia miltiorrhiza inhibits intimal hyperplasia and monocyte chemotactic protein-1 expression after balloon injury in cholesterol-fed rabbits," Journal of Cellular Biochemistry, vol. 83, no. 3, pp. 484-493, 2001.

[133] Y. J. Wu, C. Y. Hong, S. J. Lin, P. Wu, and M. S. Shiao, "Increase of vitamin $\mathrm{E}$ content in LDL and reduction of atherosclerosis in cholesterol-fed rabbits by a water-soluble antioxidant-rich fraction of Salvia miltiorrhiza," Arteriosclerosis, Thrombosis, and Vascular Biology, vol. 18, no. 3, pp. 481-486, 1998.

[134] W. Ji and B. Q. Gong, "Hypolipidemic activity and mechanism of purified herbal extract of Salvia miltiorrhiza in hyperlipidemic rats," Journal of Ethnopharmacology, vol. 119, no. 2, pp. 291-298, 2008.

[135] M. Wang, J. Liu, B. Zhou et al., "Acute and sub-chronic toxicity studies of Danshen injection in Sprague-Dawley rats," Journal of Ethnopharmacology, vol. 141, no. 1, pp. 96-103, 2012.

[136] K. Chan, A. C. T. Lo, J. H. K. Yeung, and K. S. Woo, “The effects of Danshen (Salvia miltiorrhiza) on warfarin pharmacodynamics and pharmacokinetics of warfarin enantiomers in rats," Journal of Pharmacy and Pharmacology, vol. 47, no. 5, pp. 402-406, 1995.

[137] T. Y. K. Chan, "Interaction between warfarin and danshen (Salvia miltiorrhiza)," Annals of Pharmacotherapy, vol. 35, no. 4, pp. 501-504, 2001.
[138] Z. H. Song, Z. N. Ji, C. K. Lo et al., "Chemical and biological assessment of a traditional Chinese herbal decoction prepared from Radix Astragali and Radix Angelicae Sinensis: orthogonal array design to optimize the extraction of chemical constituents," Planta Medica, vol. 70, no. 12, pp. 1222-1227, 2004.

[139] H. Zhang, S. Chen, X. Deng, X. Yang, and X. Huang, "The effects of Danggui-Buxue-Tang on blood lipid and expression of genes related to foam cell formation in the early stage of atherosclerosis in diabetic GK rats," Diabetes Research and Clinical Practice, vol. 77, no. 3, pp. 479-481, 2007.

[140] Z. W. Lin, L. Y. Zhou, and Q. L. Yi, "Effect of the Danggui Buxue decoction on blood lipid of rats with atherosclerosis," Clinical Journal of Chinese Medicine, vol. 5, no. 12, pp. 19-20, 2013.

[141] Y. Chen, Q. Chen, J. Lu, F. H. Li, Y. Y. Tao, and C. H. Liu, "Effects of Danggui Buxue decoction on lipid peroxidation and MMP-2/9 activities of fibrotic liver in rats," Chinese Journal of Integrative Medicine, vol. 15, no. 6, pp. 435-441, 2009.

[142] C. C. Wang, K. F. Cheng, W. M. Lo et al., "A randomized, doubleblind, multiple-dose escalation study of a Chinese herbal medicine preparation (Dang Gui Buxue Tang) for moderate to severe menopausal symptoms and quality of life in postmenopausal women," Menopause, vol. 20, no. 2, pp. 223-231, 2013.

[143] C. M. Koon, K. S. Woo, P. C. Leung, and K. P. Fung, "Salviae Miltiorrhizae Radix and Puerariae Lobatae Radix herbal formula mediates anti-atherosclerosis by modulating key atherogenic events both in vascular smooth muscle cells and endothelial cells," Journal of Ethnopharmacology, vol. 138, no. 1, pp. 175-183, 2011.

[144] C. C. Fong, F. Wei, Y. Chen et al., "Danshen-Gegen decoction exerts proliferative effect on rat cardiac myoblasts $\mathrm{H} 9 \mathrm{c} 2$ via MAPK and insulin pathways," Journal of Ethnopharmacology, vol. 138, no. 1, pp. 60-66, 2011.

[145] D. Wing-Shing Cheung, C. M. Koon, C. F. Ng et al., "The roots of Salvia miltiorrhiza (Danshen) and Pueraria lobata (Gegen) inhibit atherogenic events: a study of the combination effects of the 2-herb formula," Journal of Ethnopharmacology, vol. 143, no. 3, pp. 859-866, 2012.

[146] D. W. S. Cheung, C. M. Koon, E. Wat et al., "A herbal formula containing roots of Salvia miltiorrhiza (Danshen) and Pueraria lobata (Gegen) inhibits inflammatory mediators in LPS-stimulated RAW 264.7 macrophages through inhibition of nuclear factor $\kappa \mathrm{b}(\mathrm{NF} \kappa \mathrm{B})$ pathway," Journal of Ethnopharmacology, vol. 145, no. 3, pp. 776-783, 2013.

[147] D. P. Sieveking, K. S. Woo, K. P. Fung, P. Lundman, S. Nakhla, and D. S. Celermajer, "Chinese herbs Danshen and Gegen modulate key early atherogenic events in vitro," International Journal of Cardiology, vol. 105, no. 1, pp. 40-45, 2005.

[148] W. Y. Tam, P. Chook, M. Qiao et al., "The efficacy and tolerability of adjunctive alternative herbal medicine (Salvia miltiorrhiza and Pueraria lobata) on vascular function and structure in coronary patients," Journal of Alternative and Complementary Medicine, vol. 15, no. 4, pp. 415-421, 2009.

[149] C. M. Koon, C. H. Ko, X. X. Sun et al., "Gene expression profiling on the molecular action of danshen-gegen formula in a randomized placebo-controlled trial of postmenopausal women with hypercholesterolemia," Evidence-Based Complementary and Alternative Medicine, vol. 2013, Article ID 703705, 14 pages, 2013.

[150] L. Zhou, S. Wang, Z. Zhang et al., "Pharmacokinetic and pharmacodynamic interaction of Danshen-Gegen extract with 
warfarin and aspirin," Journal of Ethnopharmacology, vol. 143, no. 2, pp. 648-655, 2012.

[151] J. J. Li, J. T. Li, and J. P. Fu, "Erxian Tang-introduction of a Chinese herbal formula, clinical practice, and experimental studies," Chinese Journal of Integrative Medicine, vol. 13, no. 1, pp. 67-73, 2007.

[152] S. C. W. Sze, C. W. Ip, T. B. Ng et al., "Compatibility of multiple herbal components in Erxian Decoction, a Chinese medicinal formula, for treating osteoporosis," European Journal of Integrative Medicine, vol. 4, no. 2, pp. e187-e196, 2012.

[153] L. L. D. Zhong, Y. Tong, G. W. K. Tang et al., "A randomized, double-blind, controlled trial of a Chinese herbal formula (ErXian decoction) for menopausal symptoms in Hong Kong perimenopausal women," Menopause, vol. 20, no. 7, pp. 767-776, 2013.

[154] S. C. W. Sze, H. P. Cheung, T. B. Ng et al., "Effects of Erxian decoction, a Chinese medicinal formulation, on serum lipid profile in a rat model of menopause," Chinese Medicine, vol. 6, article 40, 2011.

[155] Y. Yang, J. Qin, B. Ke et al., "Effect of Linguizhugan decoction on hyperlipidemia rats with intermittent fasting," Journal of Traditional Chinese Medicine, vol. 33, no. 2, pp. 250-252, 2013.

[156] D. S. Chen, B. Ke, Y. J. Huang et al., "Effects of the modified Linggui Zhugan Decoction combined with short-term very low calorie diets on glycemic control in newly diagnosed type 2 diabetics," Journal of Traditional Chinese Medicine, vol. 31, no. 3, pp. 185-188, 2011.

[157] C. Lara-Castro, B. R. Newcomer, J. Rowell et al., "Effects of short-term very low-calorie diet on intramyocellular lipid and insulin sensitivity in nondiabetic and type 2 diabetic subjects," Metabolism: Clinical and Experimental, vol. 57, no. 1, pp. 1-8, 2008.

[158] H. R. Lijnen, M. Van Hul, and B. Hemmeryckx, "Caloric restriction improves coagulation and inflammation profile in obese mice," Thrombosis Research, vol. 129, no. 1, pp. 74-79, 2012.

[159] Y. Date and K. Kangawa, "Ghrelin as a starvation signal," Obesity Research and Clinical Practice, vol. 6, no. 4, pp. e263-e269, 2012.

[160] T. Liu, L. L. Yang, L. Zou et al., "Chinese medicine formula lingguizhugan decoction improves beta-oxidation and metabolism of fatty acid in high-fat-diet-induced rat model of fatty liver disease," Evidence-Based Complementary and Alternative Medicine, vol. 2013, Article ID 429738, 9 pages, 2013.

[161] Y. Li, M. Gong, and T. Konishi, "Antioxidant synergism among component herbs of traditional Chinese medicine formula, ShengMai San studied in vitro and in vivo," Journal of Health Science, vol. 53, no. 6, pp. 692-699, 2007.

[162] Q. Ni, J. Wang, E. Q. Li et al., "Study on the protective effect of Shengmai San on the myocardium in the type 2 diabetic cardiomyopathy model rat," Journal of Traditional Chinese Medicine, vol. 31, no. 3, pp. 209-219, 2011.

[163] Y. Z. Zhang, H. Y. Wu, L. W. Ren, H. S. Zhang, X. Jia, and Y. Z. Zhang, "Study on modified Shengmai Yin injection for prevention and treatment of brain impairment in endotoxin shock rats," Journal of Traditional Chinese Medicine, vol. 30, no. 4, pp. 272-277, 2010.

[164] M. Xu, G. Wang, H. Xie, Q. Huang, W. Wang, and Y. Jia, "Pharmacokinetic comparisons of schizandrin after oral administration of schizandrin monomer, Fructus Schisandrae aqueous extract and Sheng-Mai-San to rats," Journal of Ethnopharmacology, vol. 115, no. 3, pp. 483-488, 2007.
[165] L. Cao, Y. Deng, G. Ding, Z. Z. Wang, and W. Xiao, "Effects of Shengmaidan Chewable Tablets on blood lipid and hemorheology in hyperlipedemic model rats," Drugs \& Clinic, vol. 27, no. 3, pp. 214-217, 2012.

[166] H. T. Yao, Y. W. Chang, C. T. Chen, M. T. Chiang, L. Chang, and T. K. Yeh, "Shengmai San reduces hepatic lipids and lipid peroxidation in rats fed on a high-cholesterol diet," Journal of Ethnopharmacology, vol. 116, no. 1, pp. 49-57, 2008.

[167] J. Wang, X. C. Yang, F. Y. Chu et al., “The effects of Xuefu Zhuyu and Shengmai on the evolution of syndromes and inflammatory markers in patients with unstable angina pectoris after percutaneous coronary intervention: a randomised controlled clinical trial," Evidence-Based Complementary and Alternative Medicine, vol. 2013, Article ID 896467, 9 pages, 2013.

[168] Y. C. Zhang, B. J. Lu, M. H. Zhao, Y. Z. Rong, and R. M. Chen, "Effect of Shengmai injection on vascular endothelial and heart functions in patients with coronary heart disease complicated with diabetes mellitus," Chinese Journal of Integrative Medicine, vol. 14, no. 4, pp. 281-285, 2008.

[169] C. Y. Chen, L. Y. Lu, P. Chen et al., "Shengmai injection, a traditional Chinese patent medicine, for intradialytic hypotension: a systematic review and meta-analysis," Evidence-Based Complementary and Alternative Medicine, vol. 2013, Article ID 703815, 14 pages, 2013.

[170] Q. Su and Y. Li, "Interaction between warfarin and the herbal product Shengmai-Yin: a case report of intracerebral hematoma," Yonsei Medical Journal, vol. 51, no. 5, pp. 793-796, 2010.

[171] Y. Mu, J. Zhang, S. Zhang et al., "Traditional Chinese medicines $\mathrm{Wu}$ Wei Zi (Schisandra chinensis Baill) and Gan Cao (Glycyrrhiza uralensis Fisch) activate pregnane $\mathrm{X}$ receptor and increase warfarin clearance in rats," Journal of Pharmacology and Experimental Therapeutics, vol. 316, no. 3, pp. 1369-1377, 2006.

[172] E. C. H. Yip, A. M. F. Liu, J. T. Y. Wong, and Y. H. Wong, "An aqueous extract of the popular Chinese nutraceutical Kwei Ling Ko (Tortoise shell-Rhizome Jelly) activates the PPAR $\gamma$ pathway and down-regulates the NFKB activity," Phytomedicine, vol. 12, no. 10, pp. 748-759, 2005.

[173] J. H. Wu, Q. H. Wang, F. Li et al., "Suppression of dietinduced hypercholesterolemia by turtle jelly, a traditional Chinese functional food, in rats," Evidence-Based Complementary and Alternative Medicine, vol. 2012, Article ID 320304, 15 pages, 2012.

[174] L. Zhang, L. Zhu, Y. Wang et al., "Characterization and quantification of major constituents of Xue Fu Zhu Yu by UPLC-DADMS/MS," Journal of Pharmaceutical and Biomedical Analysis, vol. 62, pp. 203-209, 2012.

[175] J. J. Lee, W. H. Hsu, T. L. Yen et al., "Traditional Chinese medicine, Xue-Fu-Zhu-Yu decoction, potentiates tissue plasminogen activator against thromboembolic stroke in rats," Journal of Ethnopharmacology, vol. 134, no. 3, pp. 824-830, 2011.

[176] F. Y. Chu, J. Wang, K. W. Yao, and Z. Z. Li, "Effect of Xuefu Zhuyu Capsule on the symptoms and signs and health-related quality of life in the unstable angina patients with bloodstasis syndrome after percutaneous coronary intervention: a randomized controlled trial," Chinese Journal of Integrative Medicine, vol. 16, no. 5, pp. 399-405, 2010.

[177] X. M. Yu, G. H. Yang, and P. Li, "Mechanism of lowering blood lipids of Xuefuzhuyu decoction in patients with hyperlipidemia," Liaoning Journal of Traditional Chinese Medicine, vol. 2, pp. 289-291, 2014. 
[178] C. Dong, W. Huang, Z. H. Geng, and S. Gao, "Effects of Xuefu Zhyyu decoction on intercellular adhesion molecule and lipid peroxidation of experimental atherosclerosis rats," Journal of Hebei University (Natural Science Edition), vol. 32, no. 6, pp. 650-654, 2012.

[179] Z. H. Geng, B. D. Shou, H. Liu, Y. B. Yang, and J. J. Ren, "Effect of Xuefu Zhuyu decoction on the expression of blood-lipid and vasoactive substances in the rats with atherosclerosis," Journal of Hebei University (Natural Science Edition), vol. 31, no. 3, pp. 304-308, 2011.

[180] D. Y. Yu, K. B. Wei, and X. D. Wo, "Clinical and experimental study of Xuefu Zhuyu Tang in treating Qi stagnation and the blood stasis type of hyperlipidemia," Chinese Journal of Modern Developments in Traditional Medicine, vol. 8, no. 10, pp.601-603, 1988.

[181] X. Song, J. Wang, P. Wang, N. Tian, M. Yang, and L. Kong, "1H NMR-based metabolomics approach to evaluate the effect of Xue-Fu-Zhu-Yu decoction on hyperlipidemia rats induced by high-fat diet," Journal of Pharmaceutical and Biomedical Analysis, vol. 78-79, pp. 202-210, 2013.

[182] L. Liu, Y. Cheng, and H. Zhang, "Phytochemical analysis of antiatherogenic constituents of Xue-Fu-Zhu-Yu-Tang using HPLCDAD-ESI-MS," Chemical and Pharmaceutical Bulletin, vol. 52, no. 11, pp. 1295-1301, 2004.

[183] M. R. Wang and M. X. Han, "Advances in researches on visceral pathogenesis of hyperlipidemia," Chinese Journal of Basic Medicine in Traditional Chinese Medicine, vol. 11, no. 4, pp. 316-319, 2005.

[184] Y. J. Wu, Z. Wang, and Y. F. Wang, "A brief study of relationship between turbid-phlegm and hyperlipidaemia," Clinical Journal of Traditional Chinese Medicine, vol. 16, no. 3, pp. 201-202, 2004.

[185] Q. F. Xian, Y. Liu, W. Zou, and J. Wang, "Research progress of Chinese medicine treatment of HAART-related hyperlipidemia," Chinese Journal of Chinese Materia Medica, vol. 38, no. 15, pp. 2530-2533, 2013.

[186] Expert Panel on Detection, Evaluation, and Treatment of High Blood Cholesterol in Adults, "Executive summary of the third report of the National Cholesterol Education Program (NCEP) expert panel on detection, evaluation, and treatment of high blood cholesterol in adults (adult treatment panel III)," Journal of the American Medical Association, vol. 285, no. 19, pp. 24862497, 2001.

[187] T. A. Jacobson, M. Miller, and E. J. Schaefer, "Hypertriglyceridemia and cardiovascular risk reduction," Clinical Therapeutics, vol. 29, no. 5, pp. 763-777, 2007.

[188] M. J. Wilkinson, L. J. Laffin, and M. H. Davidson, "Overcoming toxicity and side-effects of lipid-lowering therapies," Best Practice \& Research Clinical Endocrinology \& Metabolism, vol. 28, no. 3, pp. 439-452, 2014.

[189] C. R. Harper and T. A. Jacobson, "The broad spectrum of statin myopathy: from myalgia to rhabdomyolysis," Current Opinion in Lipidology, vol. 18, no. 4, pp. 401-408, 2007.

[190] E. Bruckert, G. Hayem, S. Dejager, C. Yau, and B. Bégaud, "Mild to moderate muscular symptoms with high-dosage statin therapy in hyperlipidemic patients-the PRIMO study," Cardiovascular Drugs and Therapy, vol. 19, no. 6, pp. 403-414, 2005.

[191] S. Wang, Y. Hu, W. Tan et al., "Compatibility art of traditional Chinese medicine: from the perspective of herb pairs," Journal of Ethnopharmacology, vol. 143, no. 2, pp. 412-423, 2012.

[192] X. Liu, W. Y. Wu, B. H. Jiang, M. Yang, and D. A. Guo, "Pharmacological tools for the development of traditional
Chinese medicine," Trends in Pharmacological Sciences, vol. 34, no. 11, pp. 620-628, 2013.

[193] H. Feng, W. Chen, and C. Zhu, "Pharmacokinetics study of bioadhesive tablet of Panax notoginseng saponins," International Archives of Medicine, vol. 4, no. 1, article 18, 2011.

[194] X. Y. Guo, J. Liu, J. Liu et al., "Use of traditional Chinese medicine in Chinese patients with coronary heart disease," Biomedical and Environmental Sciences, vol. 26, no. 4, pp. 303310, 2013.

[195] A. Dasgupta, "Effect of herbal remedies on clinical laboratory tests," in Accurate Results in the Clinical Laboratory, A. Dasgupta and J. L. Sepulveda, Eds., Chapter 7, pp. 75-92, Elsevier, San Diego, Calif, USA, 2013.

[196] K. L. Miller, R. S. Liebowitz, and L. K. Newby, “Complementary and alternative medicine in cardiovascular disease: a review of biologically based approaches," American Heart Journal, vol. 147, no. 3, pp. 401-411, 2004.

[197] M. L. Kashyap, M. E. McGovern, K. Berra et al., "Long-term safety and efficacy of a once-daily niacin/lovastatin formulation for patients with dyslipidemia," The American Journal of Cardiology, vol. 89, no. 6, pp. 672-678, 2002.

[198] E. Chan, M. Tan, J. Xin, S. Sudarsanam, and D. E. Johnson, "Interactions between traditional Chinese medicines and Western therapeutics," Current Opinion in Drug Discovery and Development, vol. 13, no. 1, pp. 50-65, 2010.

[199] H. Wu, J. Bei, and J. Guo, "Chinese herbal medicine for treatment of dislipidemia," Journal of Geriatric Cardiology, vol. 6, no. 2, pp. 119-125, 2009.

[200] J. C. Li, X. Y. Cheng, J. Gu, and R. Tan, "The effects of GegenDanshen prescription on the lipid metabolism in hyperlipidemia rats," Journal of Southwest University for Nationalities: Natural Science Edition, vol. 36, no. 6, pp. 926-924, 2012. 

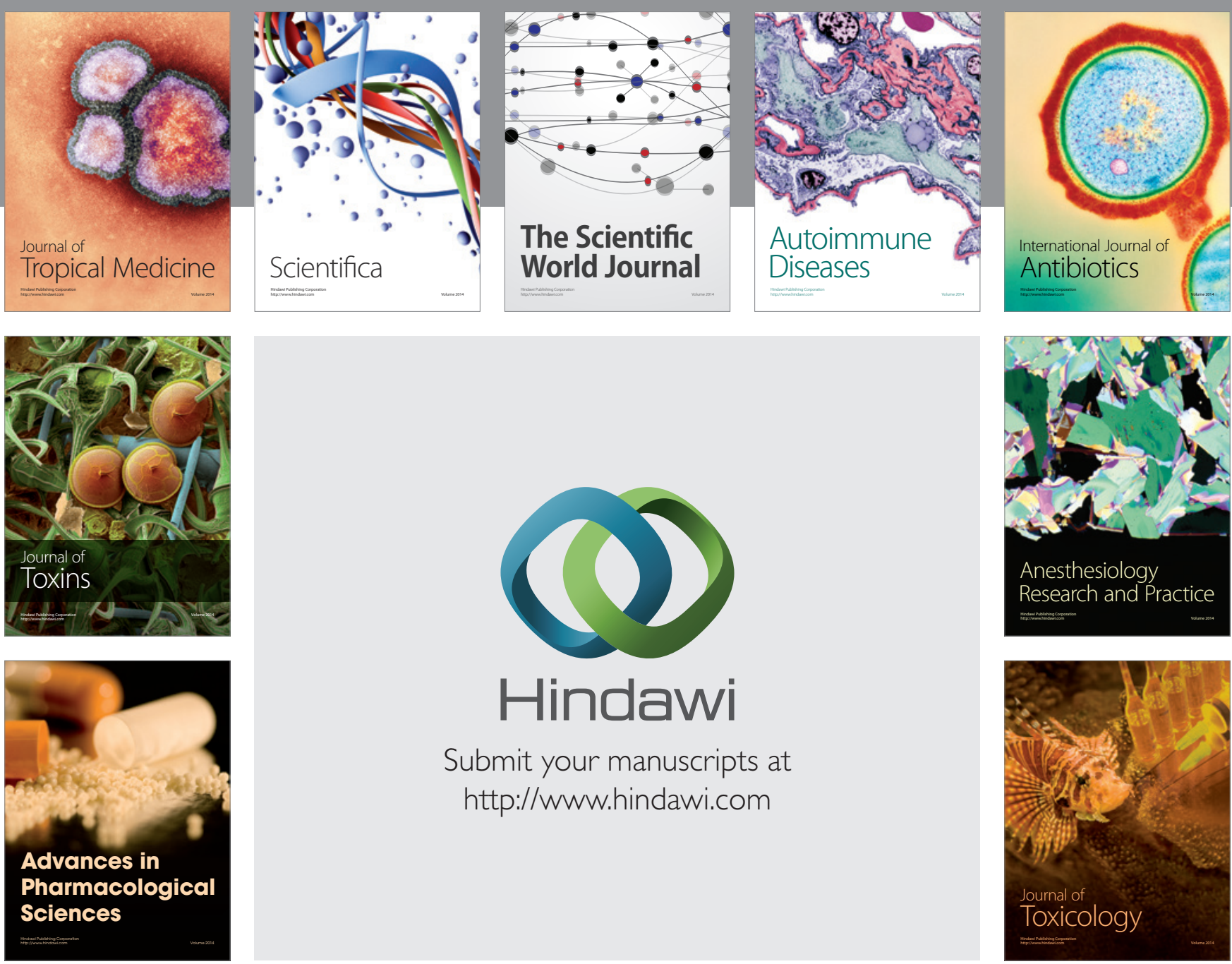

\section{Hindawi}

Submit your manuscripts at

http://www.hindawi.com
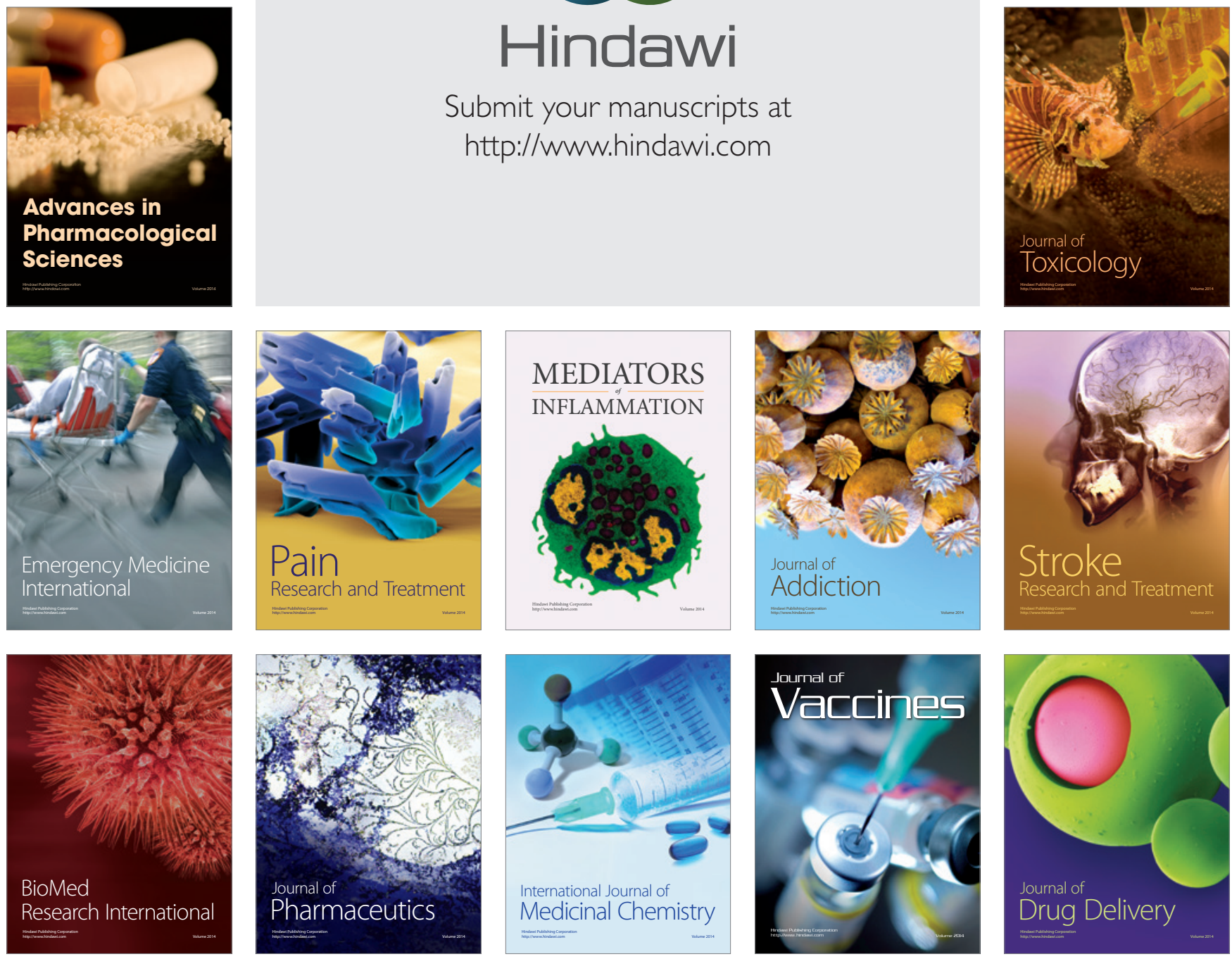